ARTICLE

\title{
WSX1 act as a tumor suppressor in hepatocellular carcinoma by downregulating neoplastic PD-L1 expression
}

Man Wu ${ }^{1,2}$, Xueqing $\mathrm{Xia}^{2}$, Jiemiao Hu (i) ${ }^{2}$, Natalie Wall Fowlkes (i) ${ }^{3} \&$ Shulin Li (i) ${ }^{2 凶}$

WSX1, a receptor subunit for IL-27, is widely expressed in immune cells and closely involved in immune response, but its function in nonimmune cells remains unknown. Here we report that WSX1 is highly expressed in human hepatocytes but downregulated in hepatocellular carcinoma (HCC) cells. Using NRAS/AKT-derived spontaneous HCC mouse models, we reveal an IL-27-independent tumor-suppressive effect of WSX1 that largely relies on CD8 ${ }^{+}$ T-cell immune surveillance via reducing neoplastic PD-L1 expression and the associated CD8 ${ }^{+}$T-cell exhaustion. Mechanistically, WSX1 transcriptionally downregulates an isoform of PI3K-PI3K $\delta$ and thereby inactivates AKT, reducing AKT-induced GSK3 $\beta$ inhibition. Activated GSK3 $\beta$ then boosts PD-L1 degradation, resulting in PD-L1 reduction. Overall, we demonstrate that WSX1 is a tumor suppressor that reinforces hepatic immune surveillance by blocking the PI3Kס/AKT/GSK3//PD-L1 pathway. Our results may yield insights into the host homeostatic control of immune response and benefit the development of cancer immunotherapies.

\footnotetext{
${ }^{1}$ Liver Cancer Institute \& Key Laboratory of Carcinogenesis and Cancer Invasion, Zhongshan Hospital, Fudan University, Shanghai 200032, PR China. ${ }^{2}$ Department of Pediatrics-Research, The University of Texas MD Anderson Cancer Center, Houston, TX 77030, USA. ${ }^{3}$ Department of Veterinary Medicine and Surgery, The University of Texas MD Anderson Cancer Center, Houston, TX 77030, USA. ${ }^{凶}$ email: sli4@mdanderson.org
} 
epatocellular carcinoma (HCC) is one of the most common malignancies worldwide and ranks as the fifth leading cause of cancer-related mortality ${ }^{1-3}$. The development of HCC is a multifactorial process in which genetic and epigenetic alterations in regulatory genes result in the activation of oncogenes and inactivation of tumor suppressor genes ${ }^{4}$. Unlike most other malignancies, HCC is an inflammation- and immunerelated tumor that arises almost exclusively in an inflamed fibrotic or cirrhotic setting. Significantly, a tightly controlled immunological network is designed to detect and eliminate transformed cells, but this process is frequently dysregulated in $\mathrm{HCC}^{5}$. Recent breakthroughs in immunotherapy targeting immune checkpoints have substantially improved HCC patient survival ${ }^{6,7}$, further supporting the crucial contribution of immune dysregulation to HCC development. However, so far, the mechanism by which HCC cells induce immunotolerance and escape from host homeostatic immunosurveillance remains unclear.

Programmed death ligand-1 (PD-L1) is a critical immune checkpoint protein whose engagement with its receptor, programmed death 1 (PD-1), on T cells activates co-inhibitory signaling to suppress effector T-cell function ${ }^{8,9}$. The physiological role of the PD-L1/PD-1 axis is maintaining the balance between peripheral tolerance and autoimmunity, but cancer cells hijack this process to escape from host immune surveillance ${ }^{10}$. Cancer cells dodge immune elimination through their expression of PD$\mathrm{L} 1$, which interacts with $\mathrm{PD}-1$ expressed on $\mathrm{T}$ cells to induce immunodepression ${ }^{11,12}$. Over the past decade, blockade of the PD-L1/PD-1 axis has shown remarkable clinical responses in a variety of advanced cancers, including $\mathrm{HCC}^{6,7,13}$. Nivolumab and durvalumab, inhibitors targeting PD-L1/PD-1, yielded promising outcomes in phase II clinical trials and were granted accelerated approval by the US Food and Drug Administration as a secondline treatment for $\mathrm{HCC}^{6,13-15}$. These encouraging clinical benefits highlight the critical role of the PD-L1/PD-1 axis in HCC pathogenesis. In physiological conditions, PD-L1 expression is under stringent control to prevent immune overreaction while maintaining a proper level of immune defense. However, this control system is often destroyed in cancer, resulting in PD-L1 dysregulation, which subsequently initiates PD-L1/PD-1 axis-mediated immune evasion and promotes tumor formation. Nonetheless, little is known about the mechanism underlying the homeostatic control of PD-L1 expression and its dysregulation in cancer. Understanding the multifaceted control of PD-L1 would facilitate the development of more effective therapeutic strategies.

WSX1, homologous to the IL- 12 receptor $\beta 2$ chain, is a class I cytokine receptor, which consists of a single transmembrane domain, a WSX1 signature motif, a box 1 motif in its intracellular region, and 7 potential $\mathrm{N}$-glycosylation sites in its extracellular domain ${ }^{16}$. WSX1, together with gp130, constitutes a functional signal-transducing receptor for IL-27; lack of either subunit attenuates IL-27-mediated signaling ${ }^{17}$. WSX1 was previously thought to be expressed exclusively in immune cells, mediating IL-27-dependent pro-inflammatory or antiinflammatory immune responses ${ }^{17,18}$. However, we and others previously demonstrated that WSX1 is also expressed in multiple types of epithelial tumor cells, including breast tumors, melanomas, and lung carcinomas ${ }^{19-21}$. The presence of WSX1 sensitized IL-27-independent natural killer (NK) cellmediated antitumor surveillance in breast tumors ${ }^{20}$ and inhibited melanoma growth in an IL-27-dependent manner ${ }^{19}$. In addition, a recent study reported that WSX1 deficiency in mice promoted the oncogenic properties of mutant $\mathrm{p} 53^{22}$, indicating a tumor-suppressive role of WSX1. These published biological functions of WSX1 largely depend on the presence of its ligand IL-27.
In this study, we reveal an IL-27-independent biological function of WSX1 as a potential HCC suppressor, a function that relies primarily on WSX1's regulation of $\mathrm{CD}^{+} \mathrm{T}$ cell-mediated adaptive immunity. In brief, we use multiple human tissue microarrays, spontaneous HCC mouse models, and current immune and molecular profiling tools to show that WSX1 downregulates neoplastic PD-L1 and reduces $\mathrm{CD}^{+}$T-cell exhaustion in the tumor microenvironment, leading to inhibition of oncogene-mediated HCC formation.

\section{Results}

WSX1 is highly expressed in human normal liver tissues, and its downregulation in HCC closely correlated with poor prognosis. While the role of WSX1 has been well studied in the immune system, its expression pattern and biological function in nonimmune cells remains unknown. To clarify the physiological distribution of WSX1, we first determined WSX1 expression using a human multiple normal tissue microarray (FDA662a) with 33 different types of human normal organ tissue. In concurrence with previous reports ${ }^{16-18}$, positive expression of WSX1 was found in immune cell-enriched tissues such as spleen and lymph node, and reached a much higher level in thymus gland and bone marrow. Unexpectedly, we observed high expression of WSX1 in multiple normal human organs, such as colon, intestine, and kidney (Supplementary Fig. 1). Notably, normal human liver tissues exhibited evenly positive staining for WSX1 (Fig. 1a), most of which was uniformly distributed in the cytoplasm and cell membrane of hepatocytes. In addition, the expression level of WSX1 in liver tissues was even higher than that in immune cell-enriched tissues. Combined with our previous discoveries that WSX1-deficient mice were highly sensitive to liver inflammation ${ }^{23,24}$, we speculate that WSX1 plays a critical role in the liver.

To validate the above results and explore whether WSX1 is associated with HCC development, we further analyzed WSX1 expression in human liver tissue microarrays (BC03116a and HLiv-HCC180Sur-03), which included 130 cases of HCC, 17 normal tissue samples from healthy donors, and 103 normal tumor-adjacent liver tissues (NAT). The clinical and pathological characteristics of all 130 HCC patients are shown in Table 1. Among the $130 \mathrm{HCC}$ cases, relative survival follow-up information was available for 90 patients (HLiv-HCC180Sur-03). Since positive-staining areas exhibited similar intensity, results were quantified as the percentage of $\mathrm{WSX}^{+}$area by ImageJ software. We consistently observed an evenly diffused positive staining pattern for WSX1 in normal liver tissues (mean \pm SD, $48.97 \% \pm$ $6.64 \%$ ), mostly in hepatocytes. However, WSX1 expression was significantly lower in NAT $(39.31 \% \pm 8.58 \%, P<0.0001)$ and was the lowest in HCC tissues $(14.31 \% \pm 7.95 \%, P<0.0001$, Fig. 1b). Additionally, using the median value as a cutoff, the 90 HCC patients in HLiv-HCC180Sur-03 were manually divided into low $(n=47)$ and high $(n=43)$ WSX1 expression groups. As expected, patients with lower WSX1 expression had shorter overall survival (hazard ratio $[\mathrm{HR}]=2.460, P=0.0034$, Fig. 1c). Furthermore, we observed that WSX1 expression was lower in histological grade III $(10.31 \% \pm 5.18 \%, n=34)$, compared to its expression in grade I $(19.51 \% \pm 8.67 \%, n=21, P<0.0001)$ and grade II $(14.80 \% \pm 7.97 \%, n=73, P=0.0123)$. No significant association of WSX1 expression with age, sex, or TNM stage was found (Table 1). Collectively, these results suggested that WSX1 plays a negative role in HCC development.

WSX1 retards HCC development in vivo independently of IL27. To clarify the role of WSX1 in HCC development, we established a spontaneous HCC mouse model in immunecompetent FVB/NJ mice. Consistent with previous studies ${ }^{25,26}$, 

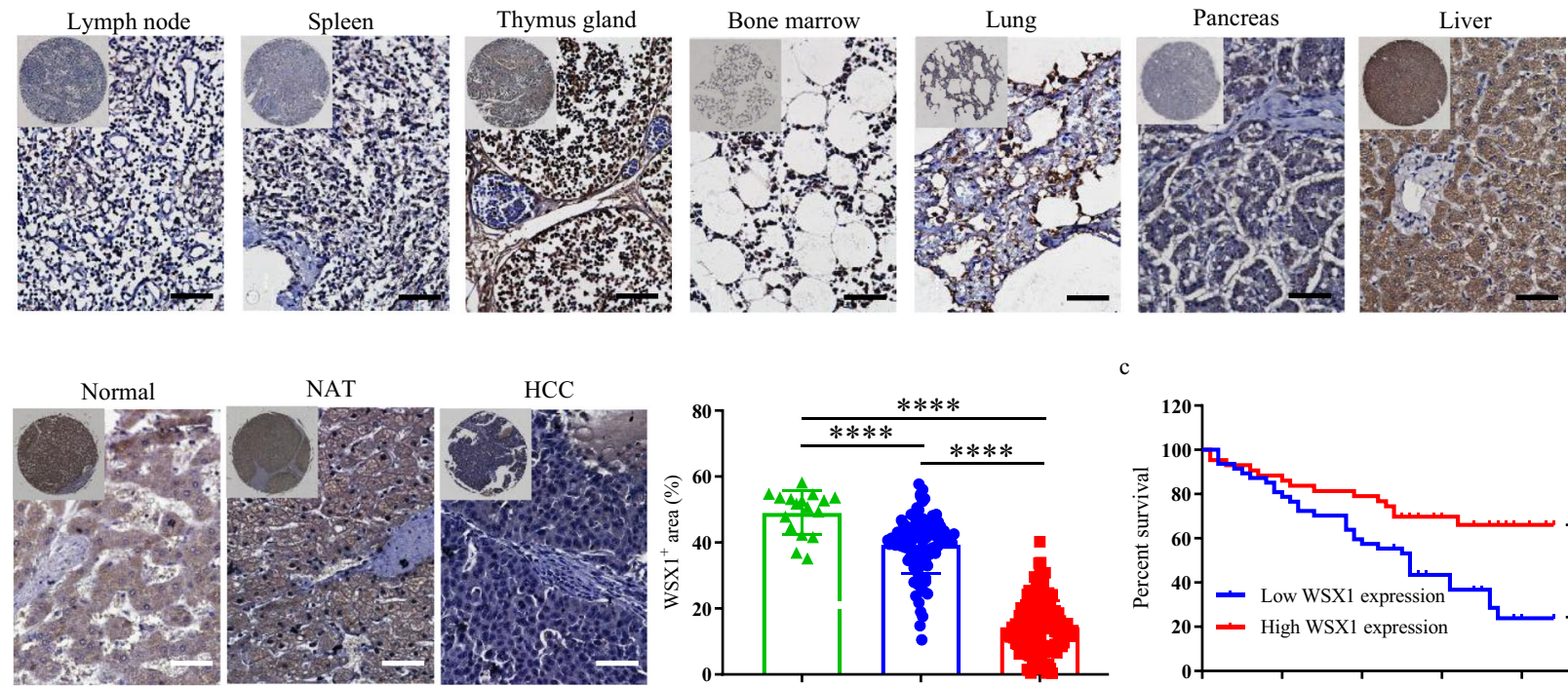

NAT

$\mathrm{HCC}$
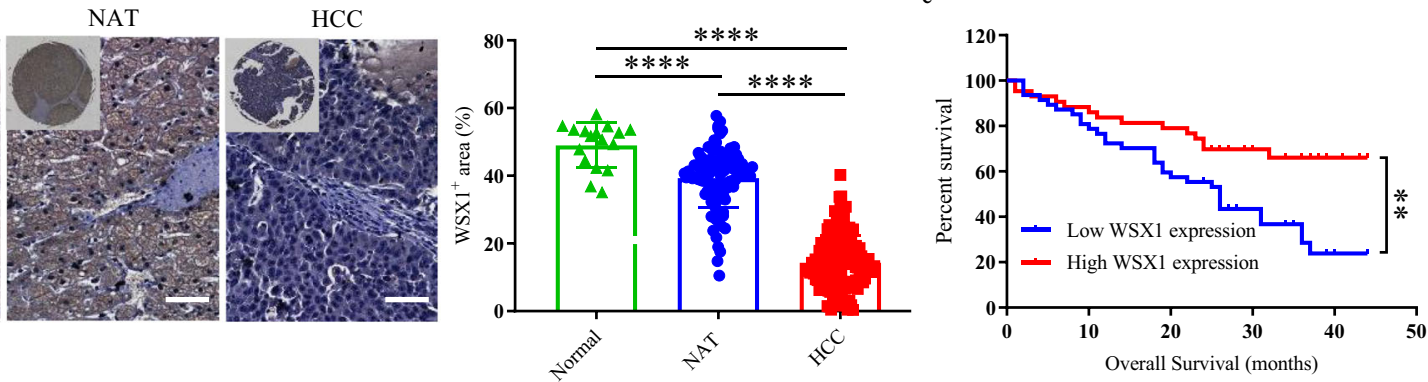

Pathological grade
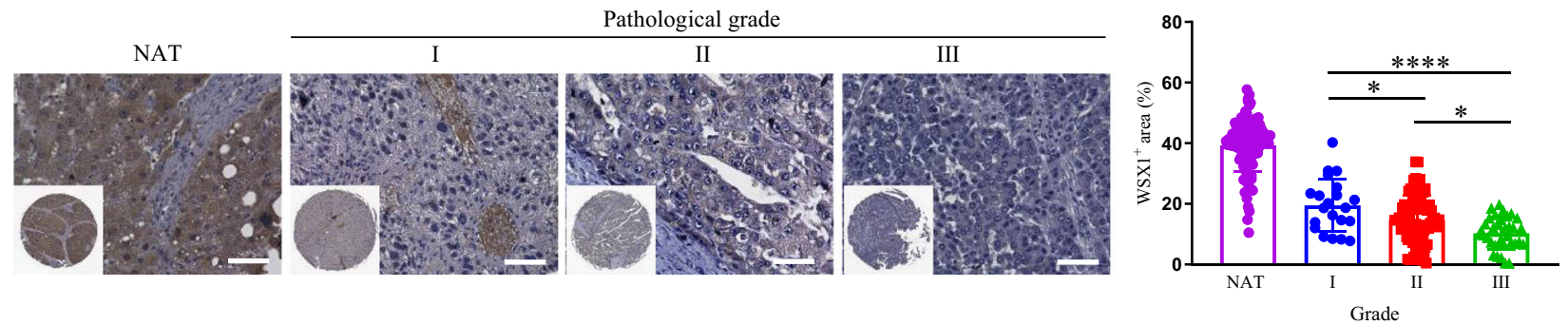

Fig. 1 WSX1 is highly expressed in normal hepatocytes, and its downregulation in HCC correlated with poor prognosis. a Immunohistochemical (IHC) staining of WSX1 in a human normal tissue microarray (TMA, FDA662a). The images shown are representatives for results of 2 individuals. $\mathbf{b}$ IHC staining of WSX1 in HCC TMAs (BC03116a and HLiv-HCC180Sur-03), including human normal liver tissue $(n=17)$, NAT ( $n=103, P<0.0001$ compared to normal liver tissue), and HCC ( $n=130, P<0.0001$ compared to both normal liver tissue and NAT) samples. Statistical analysis results are based on quantification of the percentage of WSX1+ area in each TMA tissue core. c Comparisons of the overall survival of HCC patients between low ( $n=47$ ) and high WSX1 expression group ( $n=43, P=0.0034)$. Forty HCC patients in TMA BC03116a were excluded due to lack of survival data. d WSX1 expression levels among different tumor pathological grades in HCC patients, including NAT $(n=103)$, grade I $(n=21)$, grade II ( $n=73, P=0.0321$ compared to grade I) and grade III ( $n=34, P<0.0001$ compared to grade I and $P=0.0123$ compared to gradell). Quantification and statistical analysis results are shown on the right. Scale bars, $50 \mu \mathrm{m}$. Quantitative data are presented as mean \pm SD. One-way ANOVA was used to calculate the $P$ values. Tukey-Kramer multiple comparison test was used for pairwise comparisons in the ANOVA analysis. The survival curves were analyzed by the Kaplan-Meier method, and the log-rank test was used to compare overall survival between groups. All statistical tests were two-sided. ${ }^{\star} P<0.05,{ }^{\star \star} P<0.01,{ }^{\star \star \star \star} P<0.0001$. $\mathrm{HCC}$ hepatocellular carcinoma, NAT normal tumor-adjacent liver tissue. Source data are provided as a Source Data file.

\begin{tabular}{|c|c|c|}
\hline Variable & No. of patients & $P$ value \\
\hline Age (years) & & 0.5741 \\
\hline$<60$ & 94 & \\
\hline$\geq 60$ & 36 & \\
\hline Sex & & 0.9238 \\
\hline Male & 105 & \\
\hline Female & 25 & \\
\hline Pathology Grade & & $<0.0001$ \\
\hline I & 21 & \\
\hline II & 73 & \\
\hline III & 34 & \\
\hline TNM Stage & & 0.8736 \\
\hline 1 & 10 & \\
\hline 2 & 55 & \\
\hline 3 & 56 & \\
\hline
\end{tabular}

we found that hydrodynamic injection (HDI) of NRAS/AKT oncogenes induced the development of nodular and diffuse HCC within 6 weeks with no exception (Fig. 2a). Hematoxylin and eosin (H\&E) staining revealed that neoplastic lesions occupied up to $90 \%$ of the liver parenchyma. However, with weekly HDI of plasmid DNA encoding WSX1, HCC formation was significantly retarded, with sporadic distribution of tumor nodules occupying about $20 \%$ of the liver parenchyma $(P<0.0001$, Fig. $2 b, c)$. Consistently, HDI of WSX1 caused remarkable decreases in liver weight $(P=0.0088$, Fig. 2 d), with pronounced survival extension $(\mathrm{HR}=0.2046, P=0.0217$, Fig. 2e). Moreover, consistent with our results in human HCC tissues, hepatic expression of WSX1 was decreased in oncogene-treated mice compared to untreated mice and was largely recovered following HDI of plasmids encoding WSX1 (Fig. 2k).

WSX1-1-C57BL/6 J mice were also included to establish the spontaneous HCC model, in which half doses of NRAS/AKT oncogenes were injected. Consequently, although WSX1 ${ }^{-1-}$ mice were generally viable and displayed no overt abnormalities, they 
a

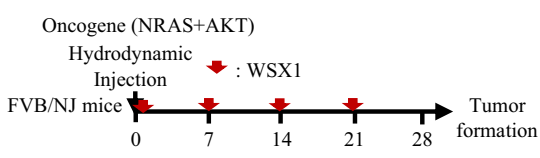

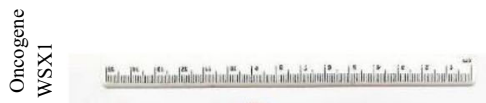
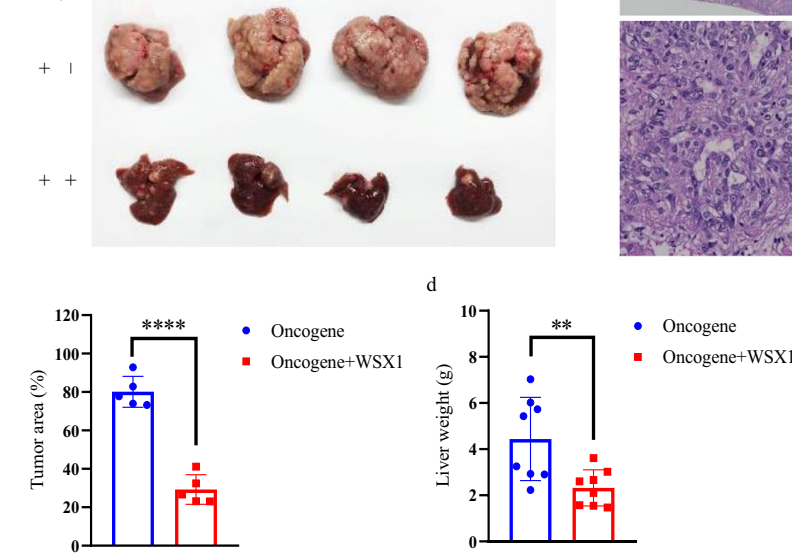

$\mathrm{f}$
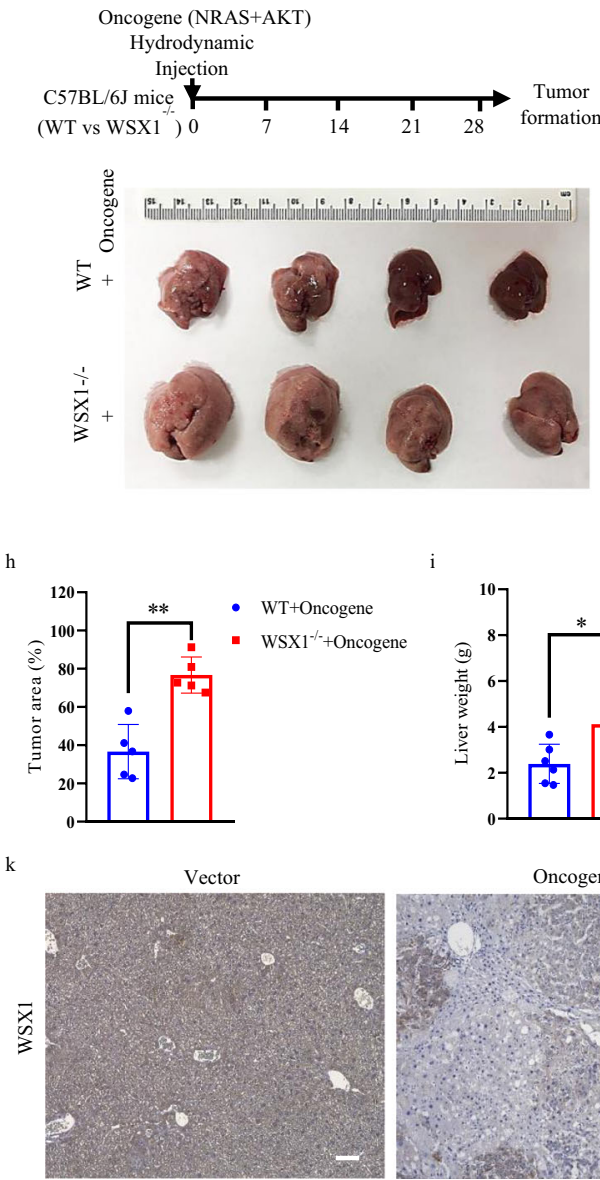

WSX1

Oncogene

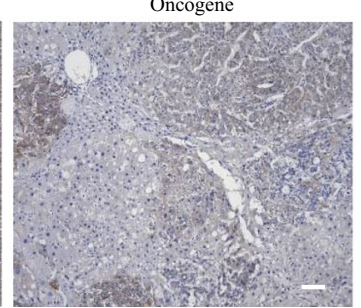

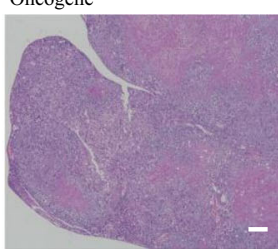
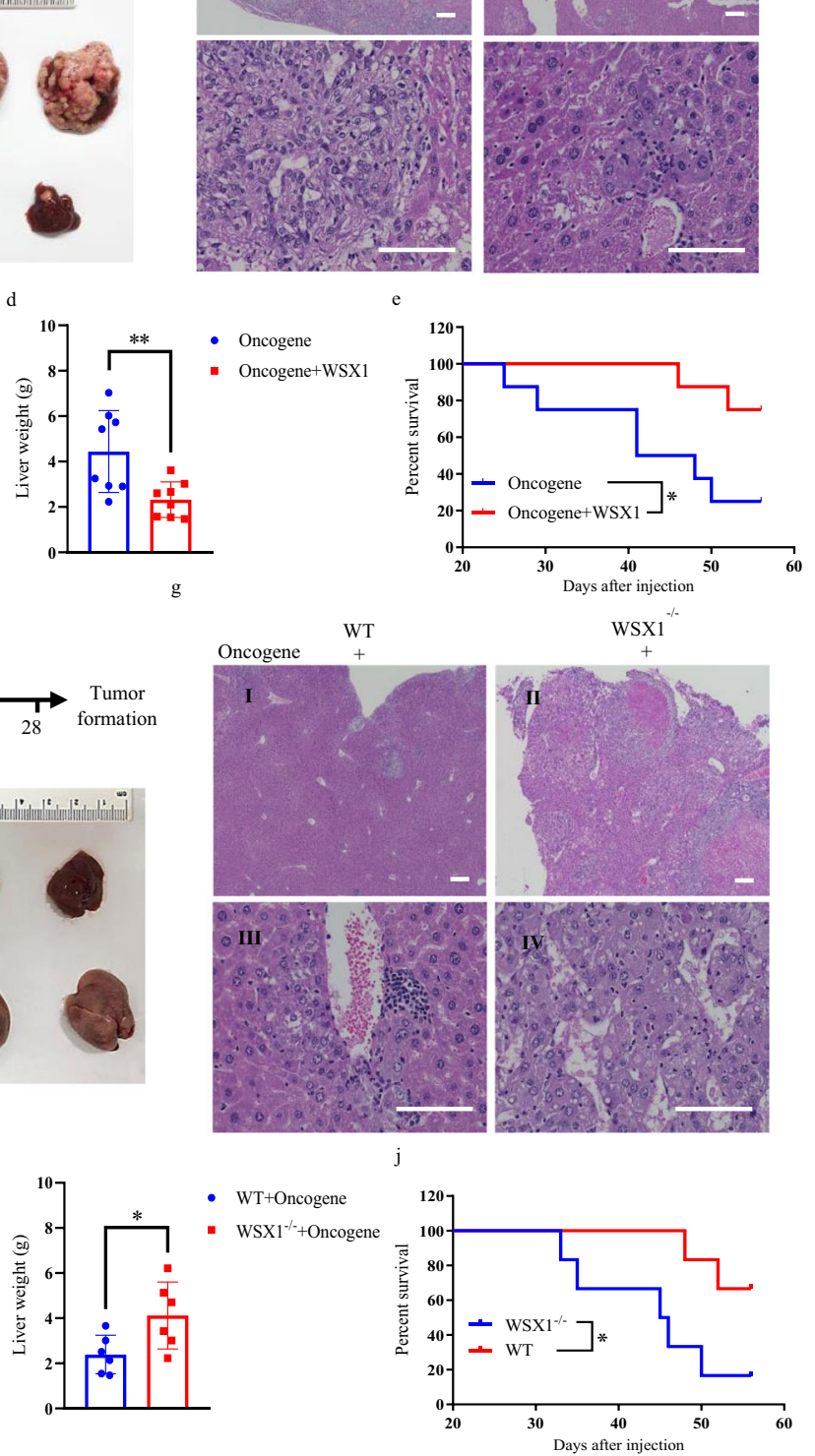

Oncogene+WSX1

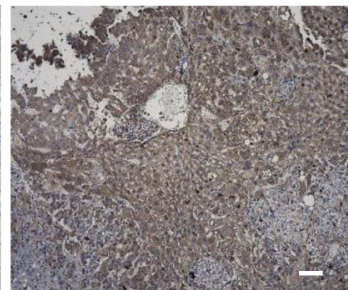

were much more susceptible to NRAS/AKT-induced oncogenesis (Fig. 2f). Specifically, compared with wild-type mice, WSX1-Imice showed earlier tumor formation, more neoplastic lesions $(P=0.0012$, Fig. $2 \mathrm{~g}, \mathrm{~h})$, increased liver weight $(P=0.0328$, Fig. $2 \mathrm{i})$, and significantly poorer survival $(\mathrm{HR}=4.821, P=$ 0.0252 , Fig. $2 \mathrm{j}$ ). Together, these results further supported the tumor-suppressive role of WSX1 in HCC development.
Furthermore, a previous study reported that the inhibitory effect of WSX1 on melanoma cell proliferation was dependent on the presence of its ligand, IL-2 $7^{19}$. Therefore, we established a spontaneous HCC mouse model in IL-27p28 $8^{-1-}$ C57BL/6J mice as well. IL-27p28 is an indispensable component of the heterodimer cytokine IL-2727. As a result, we found that IL-27p28 deficiency had no significant influence on the tumor-suppressive effect of WSX1. 
Fig. 2 WSX1 retards oncogenic NRAS/AKT-induced HCC development in vivo. a (Top) Summary of NRAS/AKT oncogene-derived spontaneous HCC mouse model in FVB/NJ mice $(n=8)$. Arrowheads represent hydrodynamic injection of WSX1 every week. (Bottom) Representative images of entire mouse livers in the oncogene and oncogene + WSX1 groups. b Comparisons of hematoxylin and eosin (H\&E) histology. c Comparisons of percentage of liver area containing preneoplastic/tumor lesions based on H\&E results $(P<0.0001)$. d Difference in liver weight between oncogene and oncogene + WSX1 groups $(P=0.0088)$. e Comparison of overall survival $(P=0.0217)$. $\mathbf{f}($ Top $)$ Summary of HCC mouse model in wild-type or WSX1-/- C57BL/6J mice $(n=6)$. (Bottom) Representative images of entire mouse livers in wild-type or WSX1-/- mice. $\mathbf{g}$ Comparisons of H\&E histology. $\mathbf{h}$ Comparisons of percentage of liver area containing preneoplastic/tumor lesions $(P=0.0012)$. i Difference in liver weight between wild-type or WSX1-/- mice $(P=$ 0.0328). $\mathbf{j}$ Comparisons of overall survival $(P=0.0252)$. $\mathbf{k}$ Expression of WSX1 in mouse liver tissues in the vector, oncogene, and oncogene + WSX 1 groups. Scale bars, $100 \mu \mathrm{m}$. All data and images are representative of 3 independent experiments. Quantitative data are presented as mean \pm SD and analyzed by two-sided Student $t$ test. The survival curves were analyzed by the Kaplan-Meier method, and the log-rank test was used to compare overall survival between groups. ${ }^{\star} P<0.05,{ }^{\star \star} P<0.01,{ }^{\star \star \star \star} P<0.0001$. Source data are provided as a Source Data file.

In the absence of IL-27, WSX1 still impaired NRAS/AKT oncogeneinduced HCC formation and improved overall survival (Supplementary Fig. 2), indicating the inhibitory function of WSX1 in HCC is independent of IL-27.

WSX1 impedes HCC development by maximizing $\mathrm{CD8}^{+} \mathrm{T}$ cell-mediated antitumor immunosurveillance. The crosstalk between tumor cells and the immune system is generally accepted as a pivotal factor in HCC development ${ }^{5}$. Considering that WSX1 had no obvious effect on HCC cell proliferation and migration in vitro (Supplementary Fig. 3b, c), and that WSX1 has long been reported to be closely connected with immunoregulation ${ }^{16-18}$, we hypothesized that the immune system might be involved in the tumor-suppressive function of WSX1. To test this hypothesis, we constructed a spontaneous HCC mouse model using immunodeficient NOD scid gamma (NSG) mice (which lack mature $\mathrm{T}$ cells, B cells, and NK cells). As expected, WSX1 failed to block NRAS/AKT oncogene-induced HCC development in these NSG mice $(P=0.99$, Supplementary Fig. 4$)$, strongly supporting our notion that an intact immune system is indispensable for the antitumor function of WSX1.

To determine how immunity contributes to the tumorsuppressive function of WSX1, we isolated intrahepatic infiltrating immune cells from entire livers obtained from spontaneous HCC mouse models and then performed cell profiling by time-offlight mass cytometry (CyTOF). PhenoGraph analysis of 34 cell markers' expression profiles identified 6 main immune cell subsets: T cells, B cells, NK cells, macrophages, dendritic cells, and other $\mathrm{CD}^{-}$cells (Fig. 3a). As shown in Fig. 3b, WSX1 had no significant effect on the proportions of these 6 cell subsets among the entire population of intrahepatic immune cells. Interestingly, about $80 \%$ of intrahepatic immune cells consisted of T-cells (Fig. 3b). Thus, our further analysis focused on T-cell clusters. As depicted, among the entire intrahepatic T-cell population, the proportions of $\mathrm{CD} 4^{+}, \mathrm{CD}^{+}, \mathrm{CD} 4^{+} \mathrm{CD} 8^{+}$double positive (DP), and $\mathrm{CD}^{-} \mathrm{CD}^{-}$double negative (DN) T-cells were similar between the oncogene and oncogene + WSX1 groups (Fig. 3c), and CD8 ${ }^{+}$T-cells constituted up to $50 \%$ of all intrahepatic T-cells in both groups. Moreover, in the T-cell panel, PhenoGraph analysis identified 13 T-cell subsets: $2 \mathrm{CD}^{+}{ }^{+}$T2 and T10), $7 \mathrm{CD}^{+}$(T3, T5, T7, T8, T9, T11, T12), 3 DP (T1, T4, T6), and $1 \mathrm{DN}$ (T13) (Fig. 3d). The expression profiles of 28 different markers on each $\mathrm{T}$-cell cluster were visualized in a heatmap (Fig. 3e). Figure $3 \mathrm{f}$ shows the proportions of each T-cell cluster among the entire intrahepatic T-cell population. Notably, WSX1 significantly increased the proportions of the T6 (DP T, $P=0.0003$ ), T9 $\left(\mathrm{CD}^{+} \mathrm{T}, P=0.0016\right)$ and T13 (DN T, $P=$ 0.0453 ) subsets, but reduced the proportions of the $\mathrm{T} 1$ $\left(\mathrm{PD}-1^{+} \mathrm{LAG} 3^{+}\right.$DP T, $\left.P=0.0114\right)$, T5 (LAG- $3^{+} \mathrm{CTLA}-4^{\mathrm{Lo}} \mathrm{CD} 8^{+}$

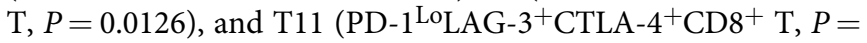
0.0216 , Fig. $3 \mathrm{f}$ ) subsets. These results indicated that WSX1 treatment predominantly affected $\mathrm{CD}^{+} \mathrm{T}$ cells. Moreover, all
$\mathrm{CD}^{+}{ }^{+} \mathrm{T}$-cell clusters, including the T1 DP T-cell cluster, that were decreased by WSX1 had high- and co-expression of PD-1, CTLA-4, and LAG-3, which are inhibitory receptors of T-cell function $^{28}$. T-cell exhaustion is often characterized by high immune inhibitory receptors co-expression (e.g., PD-1, LAG-3, CTLA-4) and progressive loss of T-cell function, including hierarchical loss of cytokine production (e.g., IL-2, IFN- $\gamma)^{29,30}$. Recent studies revealed that the HMG-box transcription factor TOX is a central regulator of T-cell exhaustion ${ }^{31-34}$.

Our independent multicolor flow cytometry experiments further validated that $\mathrm{CD} 8^{+} \mathrm{T}$ cells in the oncogene + WSX1 group had lower expression not only of T-cell exhaustion markers (PD-1: $P=0.0074$; CTLA-4: $P=0.0471$; LAG-3: $P=0.0295)$ but also of the T-cell exhaustion driver TOX $(P=0.0116$, Supplementary Fig. 5b, d). In addition, WSX1 increased the expression levels of T-cell functional markers (granzyme $\mathrm{B}$ : $P=0.0344$; perforin: $P=0.0440$; Ki67: $P=0.0418$; IL-2: $P=0.0405$; IFN- $\gamma$ : $P=0.0268$; Supplementary Fig. 5c, d). Together, the above results suggested that the presence of WSX1 could relieve CD8 ${ }^{+}$T-cell exhaustion, which might be responsible for WSX1-mediated suppression of HCC formation and progression.

To validate our hypothesis that the tumor-suppressive effect of WSX1 relies on its impact on $\mathrm{CD}^{+} \mathrm{T}$-cells, we next performed in vivo depletion of $\mathrm{CD}^{+}$and $\mathrm{CD} 4^{+}$T-cells as well as NK cells using specific antibodies. The efficiency of in vivo immune cell depletion is shown in Fig. 4a. Depletion of $\mathrm{CD}^{+}$T-cells substantially impaired WSX1-mediated inhibition of HCC development induced by oncogene attack, yielding massive nodular and diffuse liver tumors $(P=0.0002$, Fig. $4 \mathrm{~b}, \mathrm{c})$. In contrast, depletion of CD4 ${ }^{+}$T-cells or NK cells had no significant effect or only mildly increased liver weight. Accordingly, in vivo depletion of $\mathrm{CD}^{+}$T-cells almost completely abolished the WSX1-induced survival extension $(\mathrm{HR}=7.078, \quad P=0.0343$, Fig. 4d).

WSX1 relieves PD-L1/PD-1 axis-induced T-cell exhaustion by downregulating PD-L1 expression in tumor cells. Since HDI of plasmid DNA encoding WSX1 had no significant influence on WSX1 expression in intrahepatic $\mathrm{CD}^{+} \mathrm{T}$ cells (Supplementary Fig. 5d), we speculated that the regulation of WSX1 on T-cell exhaustion was mostly mediated by its effect on tumor cells. Considering that the engagement of PD-L1 with its receptor PD-1 on T-cells is the most important reason for T-cell exhaustion ${ }^{35}$, we next tested whether WSX1 reduces T-cell exhaustion by affecting PD-L1 expression in tumor cells. First, we used the human HCC cell lines SNU449 and SNU475, which have low expression levels of WSX1, to construct 2 stable cell lines with WSX1 overexpression (449WSX1 and $475^{\mathrm{WSX} 1}$ ). We also knocked down WSX1 using a CRISPR/Cas9 guiding RNA (crWSX1) in human HCC cell line SNU398, which has a relatively high expression level of WSX1 (Supplementary Fig. 3a). compared with parental cells, both the cell surface expression and total 


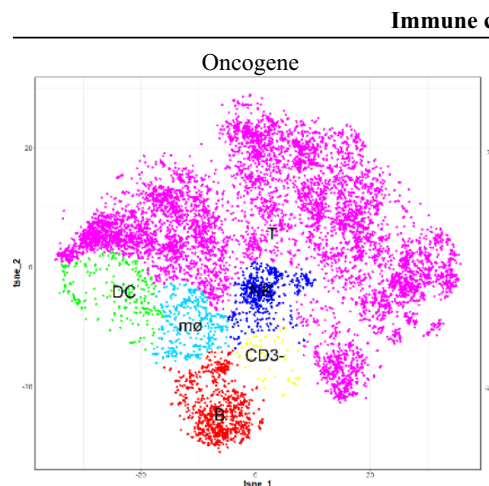

Immune cell Panel

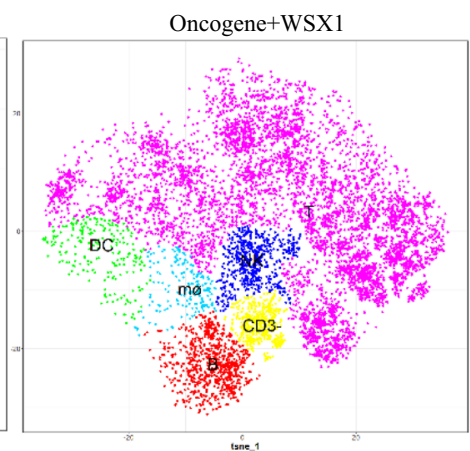

b

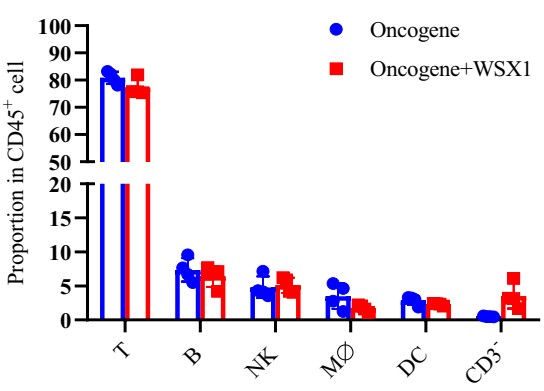

$\mathrm{d}$

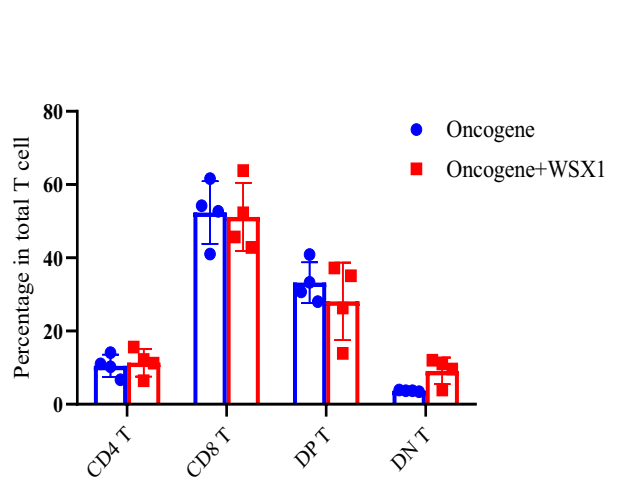

\begin{tabular}{ll} 
T cell Panel & \\
\hline Oncogene & Oncogene+WSX1
\end{tabular}
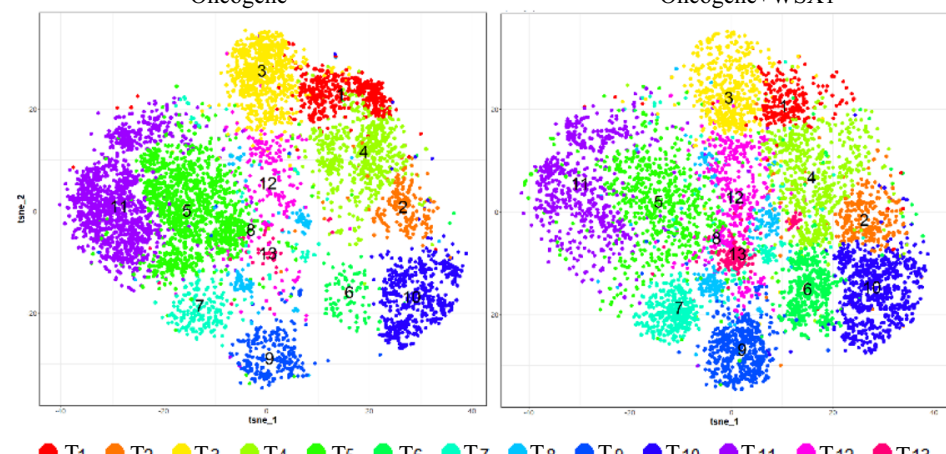

$\begin{array}{lllllllllllll}\mathrm{T}_{1} & \mathrm{~T}_{2} & \mathrm{~T}_{3} & \mathrm{~T}_{4} & \mathrm{~T}_{5} & \mathrm{~T}_{6} & \mathrm{~T}_{7} & \mathrm{~T}_{8} & \mathrm{~T}_{9} & \mathrm{~T}_{10} & \mathrm{~T}_{11} & \mathrm{~T}_{12} & \mathrm{~T}_{13}\end{array}$

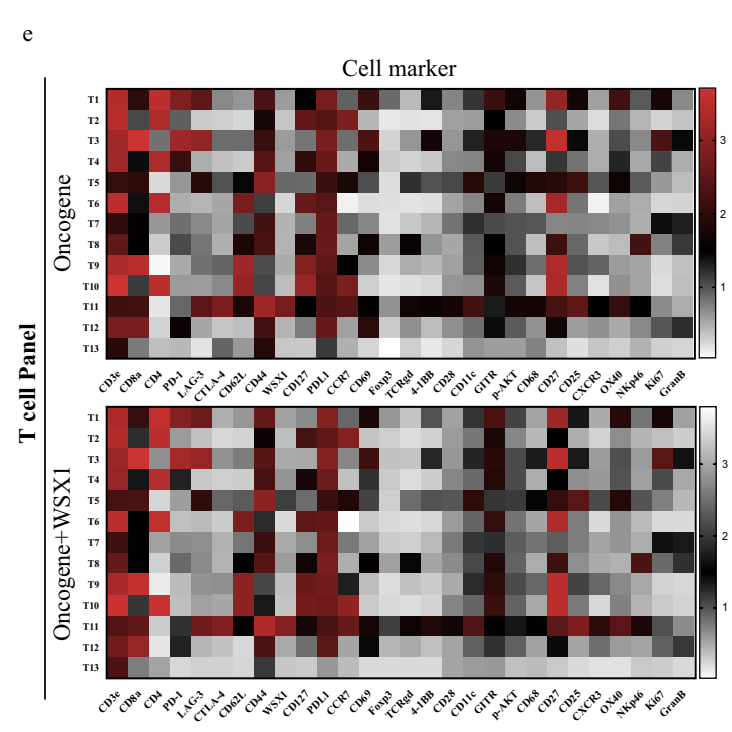

f
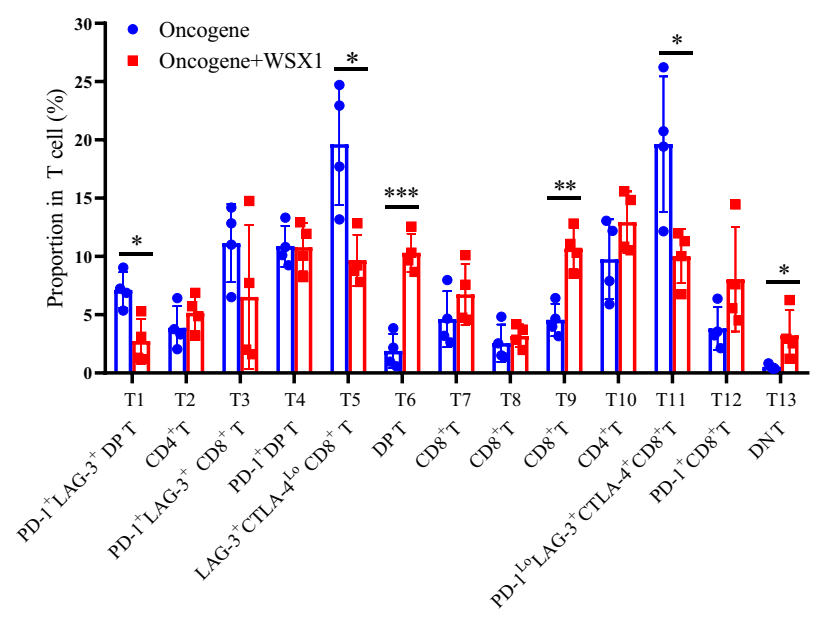

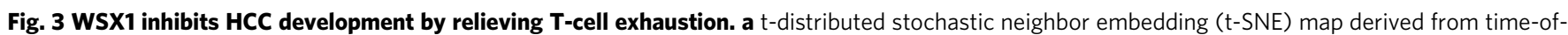
flight mass cytometry (CyTOF) analysis of intrahepatic immune cells obtained from the HCC mouse model in Fig. 2a $(n=4)$. Cells are colored by clusters identified by Rphenograph. Clusters were grouped by expression profile and manually assigned to 6 main cell subsets: T cells, B cells, NK cells, M $\phi$, DCs, and other $\mathrm{CD} 3^{-}$cells. $\mathbf{b}$ The percentage of T, B, NK, M, DC, and other CD3- cells among all intrahepatic immune cells $(n=4)$. c The proportion of CD4+, $\mathrm{CD}^{+}, \mathrm{CD} 4^{+} \mathrm{CD} 8^{+} \mathrm{DP}$, and CD4 ${ }^{-} \mathrm{CD} 8^{-} \mathrm{DN}$ T-cell subsets among total intrahepatic T cells $(n=4)$. d t-SNE map of intrahepatic T cells derived from CyTOF analysis. Rphenograph identified $13 \mathrm{~T}$-cell subsets based on expression profiles of 28 markers. e Heatmap showing expression of $28 \mathrm{~T}$-cell panel markers in 13 T-cell clusters. f Differences in the proportion of 2 CD4+ (T2 and T10), 7 CD8+ (T3, T5, T7, T8, T9, T11, T12), 3 DP (T1, T4, T6), and 1 DN (T13) T-cell clusters among total intrahepatic T cells $(n=4)$. WSX1 reduced the proportion of T1 $(P=0.0114), \mathrm{T} 5(P=0.0126)$ and T11 $(P=0.0216)$, while increased the proportion of T6 $(P=0.0003)$, T9 $(P=0.0016)$ and T13 subsets $(P=0.0453)$. All data are representative of 2 independent experiments. Quantitative data are presented as mean \pm SD and analyzed by two-sided Student $t$ test. ${ }^{\star} P<0.05,{ }^{\star \star} P<0.01,{ }^{\star \star \star} P<0.001$. NK cells natural killer cells, M $\phi$ macrophages, DCs dendritic cells, DP double positive, DN double negative. Source data are provided as a Source Data file. 

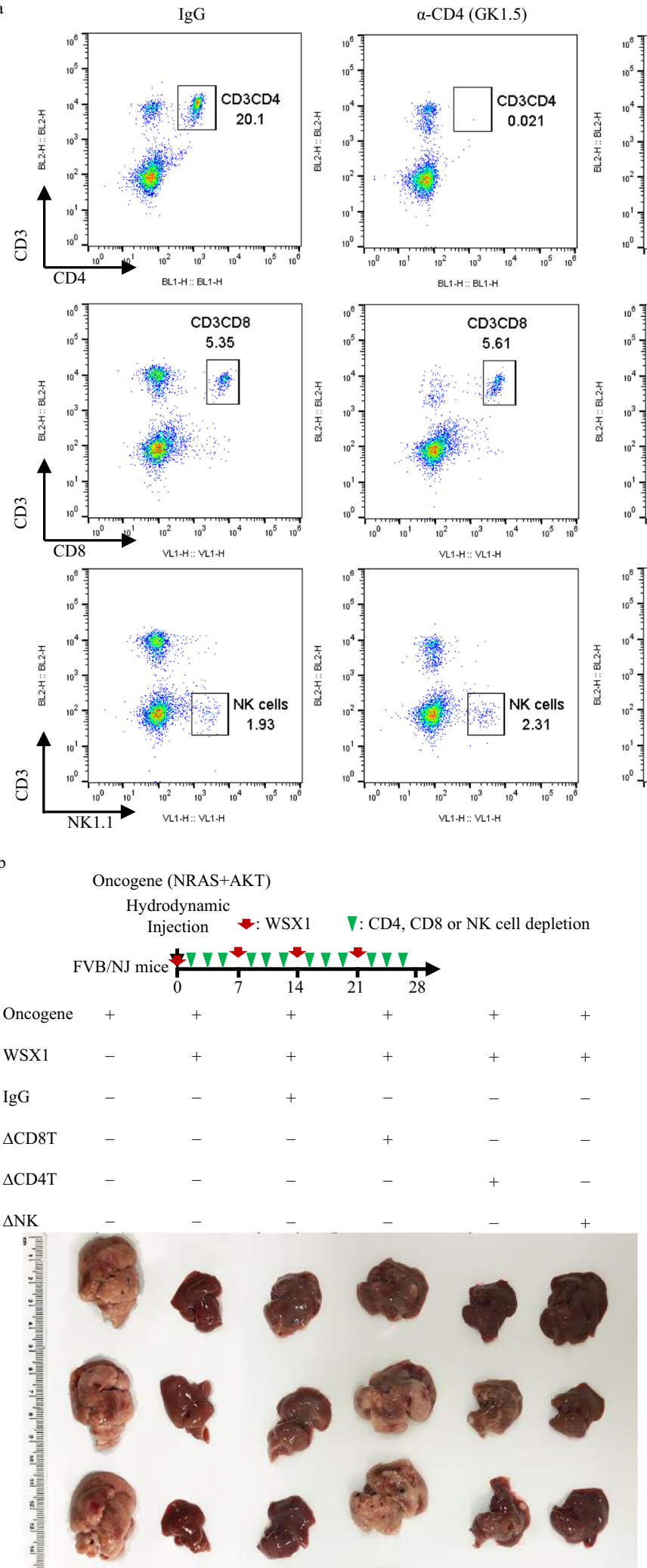

$\alpha-\operatorname{CD} 8(2.43)$
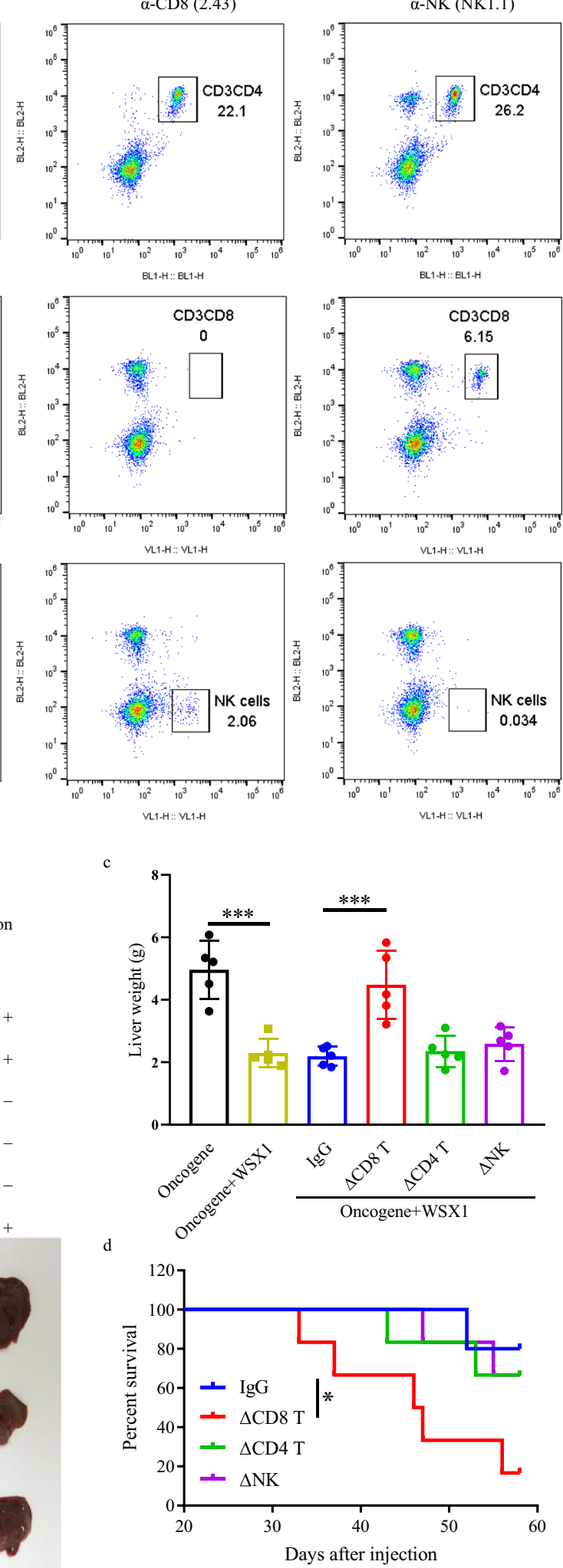

protein level of PD-L1 decreased in WSX1-overexpressing cell lines $449^{\mathrm{WSX} 1}(P<0.0001)$ and $475^{\mathrm{WSX} 1}(P=0.0002$, Fig. 5a, b), but increased in WSX1-knockdown cells $(P=0.0028$, Fig. $5 c, d)$.

To confirm the connection between WSX1 and PD-L1 in vivo, we performed CyTOF analysis of mouse hepatocytes obtained from entire livers in spontaneous HCC mouse models. Due to the distinctive characteristics of the spontaneous HCC model, there are no visible explicit boundaries at which to distinguish tumor lesions, preneoplastic areas, and normal liver tissues. And no marker is specific enough to distinguish spontaneous tumor cells from normal hepatocytes. Thus, our further studies analyzed hepatocytes as a whole. Consistent with our results in vitro, CyTOF analysis results demonstrated that WSX1 indeed decreased PD-L1 expression in hepatocytes $(P=0.0012$, Fig. $5 e, f)$, which was further confirmed by 
Fig. 4 WSX1-induced HCC regression is dependent on CD8 ${ }^{+}$T-cells. Depletion antibodies against CD8 ${ }^{+} \mathrm{T}(\alpha-\mathrm{CD} 8), \mathrm{CD} 4{ }^{+} \mathrm{T}(\alpha-\mathrm{CD} 4)$, and NK $(\alpha-N K)$ cells were used for immune-cell depletion in the spontaneous HCC mouse model. a Efficiency of in vivo immune-cell depletion was validated by flow cytometry. The gating strategy for sorting CD8 ${ }^{+}$T-cells, CD4 + T-cells, and NK cells is shown in the supplementary Fig. 8a. b Effect of WSX1 on tumor growth with or without in vivo immune-cell depletion $(n=5)$. Red arrowheads represent WSX1 injection once a week. Green arrowheads represent injection of the indicated antibodies 3 times a week. c Depletion of CD8 ${ }^{+}$T-cells impaired WSX1-mediated inhibition of HCC formation $(P=0.0002)$. d Depletion of CD8 + T-cells decreased the WSX1-induced survival extension (HR $=7.078, P=0.0338)$. All data and images are representative of 2 independent experiments. Quantitative data are presented as mean \pm SD and analyzed by One-way ANOVA analysis. Tukey-Kramer multiple comparison tests were used for pairwise comparisons in the ANOVA analysis. The survival curves were analyzed by the Kaplan-Meier method, and the log-rank test was used to compare overall survival between groups. All statistical tests were two-sided. ${ }^{\star} P<0.05,{ }^{\star \star} P<0.01,{ }^{\star \star \star} P<0.001$. Source data are provided as a Source Data file.
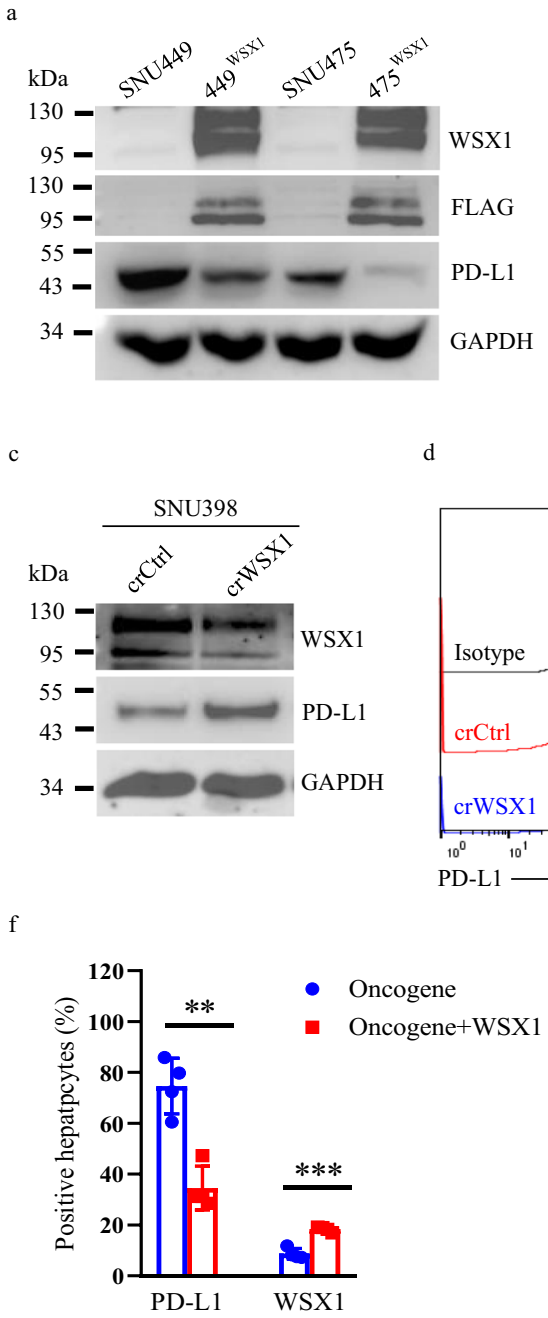

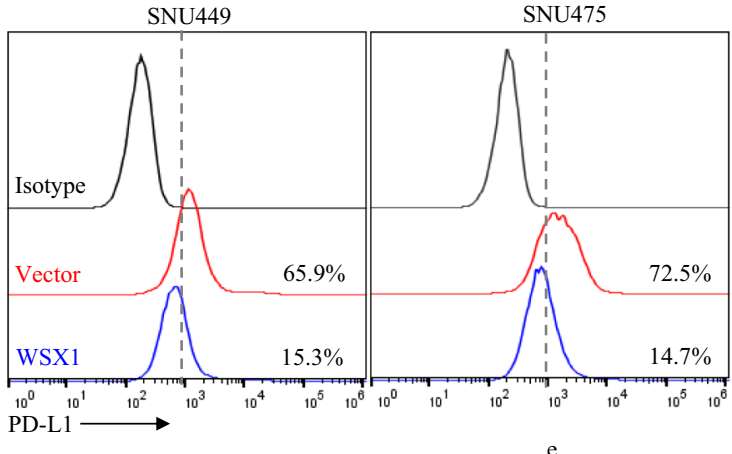

d

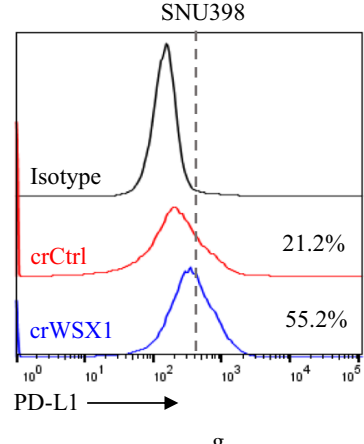

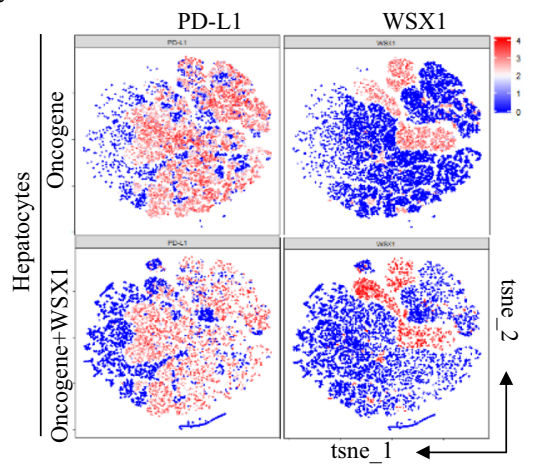
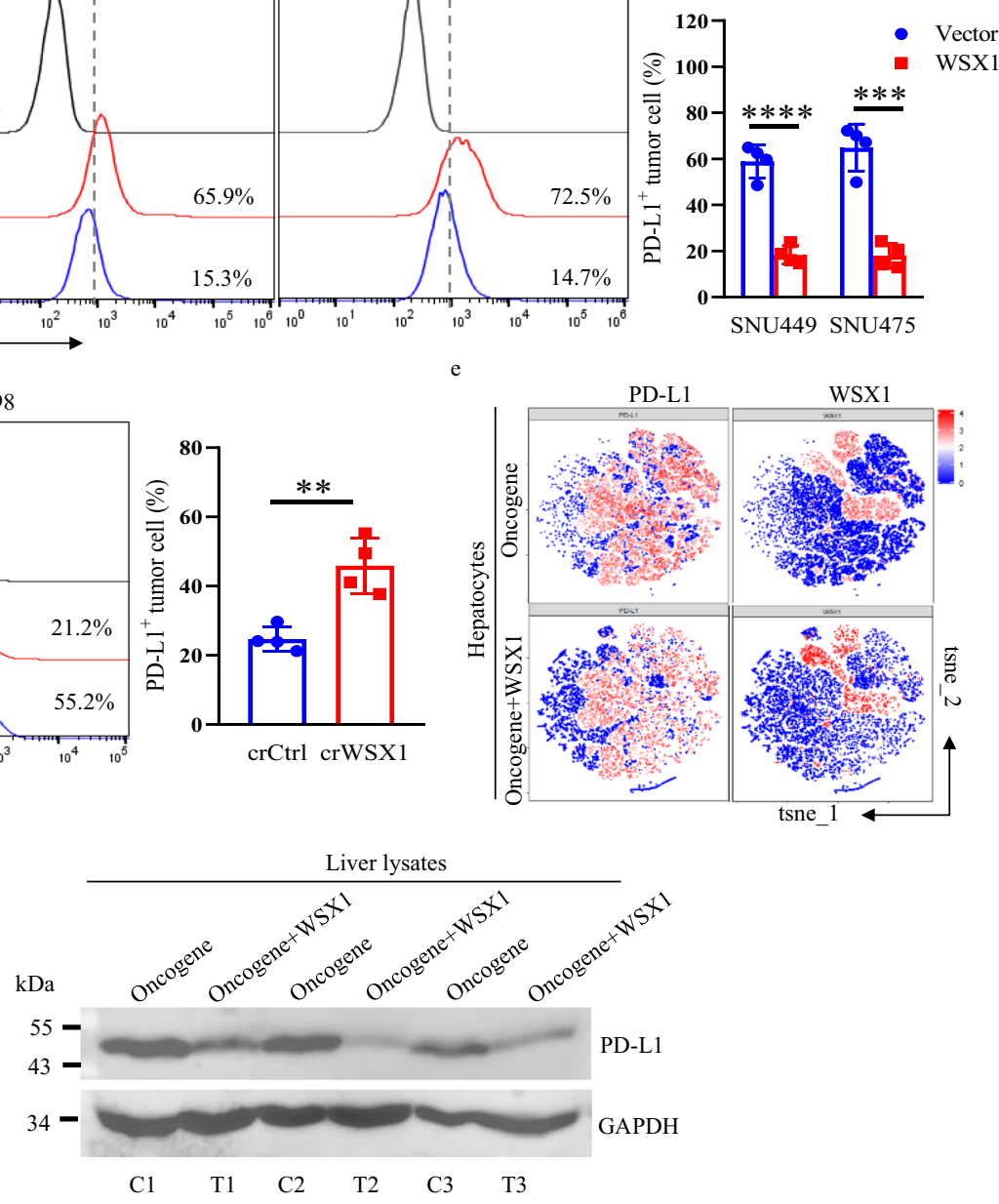

Fig. 5 WSX1 overexpression downregulates PD-L1 expression in HCC cells. a Protein levels of WSX1, FLAG, and PD-L1 analyzed by Western blotting $\mathrm{HCC}$ cell lines SNU449 and SNU475 were transfected with plasmid DNA encoding WSX1-FLAG (449WSX1 and 475 WSX1) or with vector plasmids. b Effect of WSX1 overexpression on cell surface PD-L1 expression analyzed by flow cytometry. WSX1 overexpression reduced the proportion of PD-L1+ HCC cells in both SNU449 ( $n=4$ independent experiments, $P<0.0001)$ and SNU475 cells $(P=0.0002)$. c Immunoblotting analysis of protein levels of WSX1 and PD-L1 in SNU398 cells. CRISPR/Cas9 guiding RNAs against human WSX1 (crWSX1) were used for WSX1 knockdown, and nontargeting crRNAs were added as control ( $\mathrm{crCtrl})$. d WSX1 knockdown increased the proportion of PD-L1+ HCC cells in SNU398 cells $(n=4$ independent experiments, $P=$ 0.0028). e t-SNE map derived from CyTOF analysis of mouse hepatocytes obtained from the HCC mouse model in Fig. 2a. Cells were color coded by the intensity of the expression levels of PD-L1 and WSX1. $\mathbf{f}$ Difference in the proportions of PD-L1 ${ }^{+}(n=4$ mice, $P=0.0012)$ and WSX1+ mouse hepatocytes $(P=0.0002)$ based on CyTOF analysis. Data shown are representative of 2 independent experiments. $\mathbf{g}$ Immunoblotting analysis of PD-L1 protein levels in mouse liver lysates (C1-C3 represent independent samples from "oncogene" group, T1-T3 are independent samples from "oncogene + WSX1" group). All images shown are representative of 3 independent experiments. Quantitative data are presented as mean \pm SD and analyzed by two-sided Student $t$ test. ${ }^{\star \star} P<0.01,{ }^{\star \star \star} P<0.001,{ }^{\star \star \star \star} P<0.0001$. The gating strategy for sorting PD-L1+ HCC cells is shown in Supplementary Fig. 8b. Source data are provided as a Source Data file. 
a

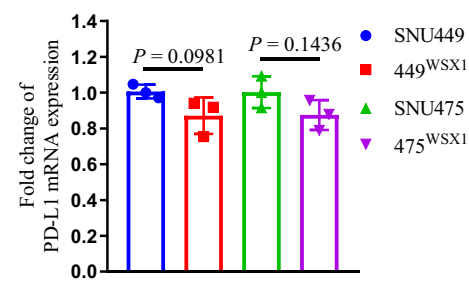

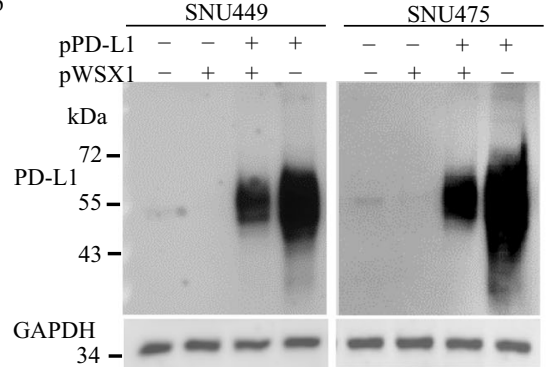
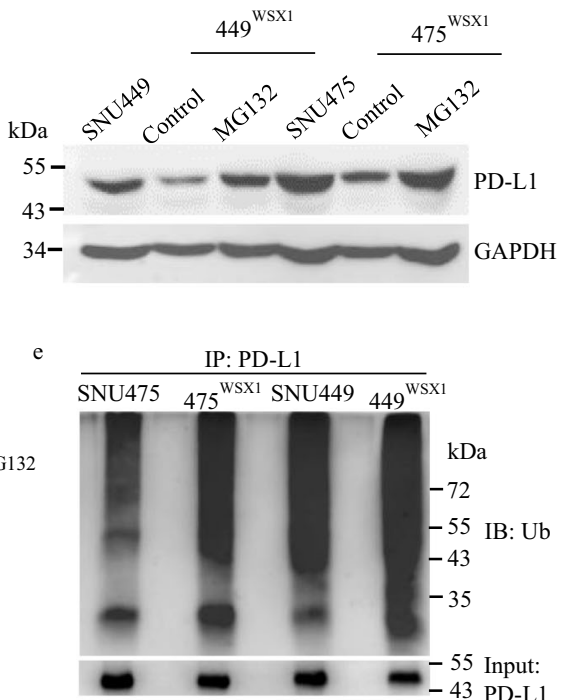

$449^{\text {WSX1 }} 475^{\text {WSX1 }}$
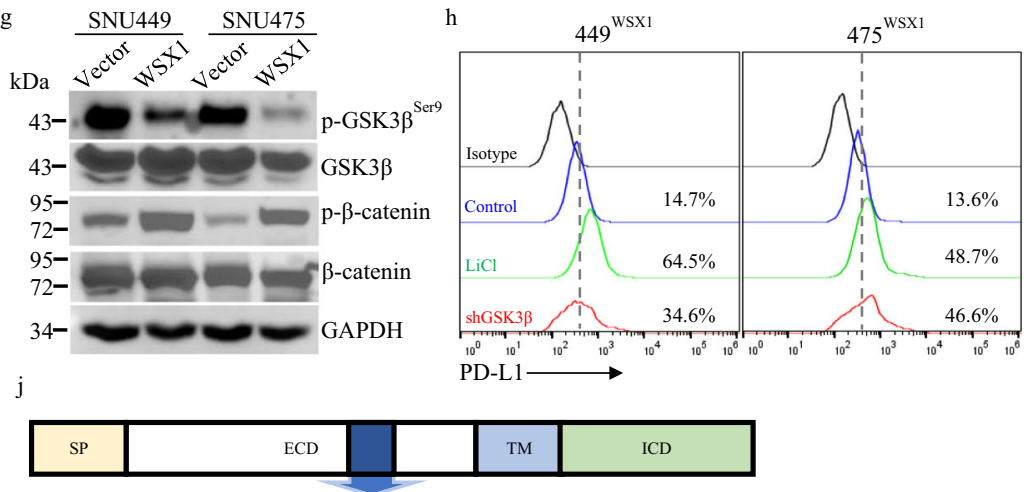

GSK3ß phosphorylation motif XSXXXTXXXSXX

S176 T180 S184

FLAG-PDL1-WT 170-DHQVLSGKTTTTNSKREEKL -190

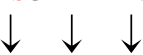

FLAG-PDL1-NP 170-DHQVLAGKTATTNAKREEKL-190

$449^{\text {WSXI }}$

4
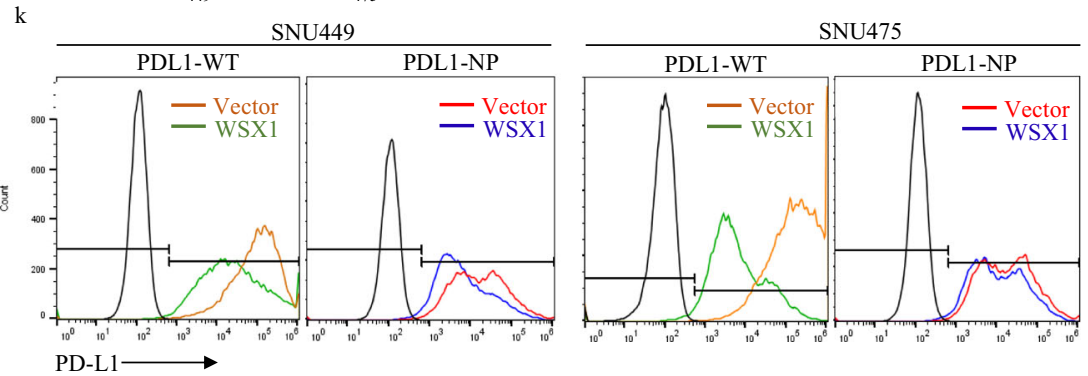

1

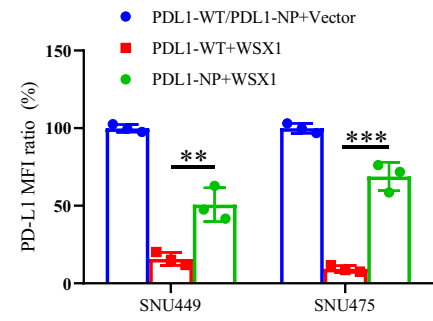

immunoblotting analysis of mouse liver lysates (Fig. 5g). Next, to further support our notion that WSX1-mediated PD-L1 downregulation could reduce $\mathrm{T}$-cell exhaustion and enhance the $\mathrm{T}$ cellmediated killing effect, we performed a co-culture assay with different ratios of activated human effector T cells (E) and tumor cells $(\mathrm{T})$. The survival rates for WSX1-overexpressing 449WSX1 (E: $\mathrm{T}=1: 1, P=0.0029 ; \mathrm{E}: \mathrm{T}=2: 1, P=0.0108)$ and $475^{\mathrm{WSX} 1}(\mathrm{E}: \mathrm{T}=$ $1: 1, P=0.0022$; $\mathrm{E}: \mathrm{T}=2: 1, P=0.0038)$ cells were less than half of the survival rates for parental cells (Supplementary Fig. 6a). In addition, T cells that were co-cultured with $449^{\mathrm{WSX} 1}(P=0.0131)$ and $475^{\mathrm{WSX} 1}(P=0.0017)$ cells had much lower expression of PD-1 (Supplementary Fig. 6b, c). Collectively, the above results verified that WSX1 can downregulate PD-L1 expression in hepatocytes and simultaneously reduce PD-L1/PD-1 axis-mediated T-cell exhaustion, ensuring the biological function of immune surveillance.

WSX1 destabilizes PD-L1 by enhancing GSK3 $\beta$-mediated PDL1 protein degradation. Motivated by our observation-downregulation of $\mathrm{PD}-\mathrm{L1}$ by WSX1-we sought to explore the underlying molecular mechanism. To this end, we first tested whether WSX1 participated in the transcriptional regulation of PD-L1. Interestingly, WSX1 had no significant effect on PD-L1 mRNA levels in either SNU449 or SNU475 cells (Fig. 6a), implying that the regulation of PD-L1 by WSX1 is at the protein level. To validate this notion, we transfected HCC cells with 
Fig. 6 WSX1 destabilizes PD-L1 by boosting GSK3ß-mediated PD-L1 degradation. a qRT-PCR assay revealed the influence of WSX1 on PD-L1 mRNA expression level ( $n=3$ independent experiments). $\mathbf{b}$ Influence of WSX1 on the expression of exogenous PD-L1-FLAG. SNU449 and SNU475 cells were transfected with plasmid DNA encoding PD-L1-FLAG with or without WSX1 co-transfection and analyzed by Western blotting. $\mathbf{c}$ Immunoblot analysis of PD-L1 protein levels after treatment with proteasome inhibitor MG132 for $12 \mathrm{~h}$. d Effect of MG132 on cell surface PD-L1 expression examined by flow cytometry. MG132 inhibited WSX1-mediated PD-L1 reduction in both SNU449 ( $n=5$ independent experiments, $P=0.0027)$ and SNU475 cells $(P=$ 0.0362). e Influence of WSX1 on PD-L1 ubiquitination. Cells were pretreated with MG132 for $12 \mathrm{~h}$, and cellular PD-L1 protein was pulled down by specific PD-L1 antibodies. PD-L1 ubiquitination was analyzed by anti-ubiquitin antibodies. $\mathbf{f}$ Impact of WSX1 on half-life of PD-L1 protein in HCC cells. HCC cells were treated with $25 \mathrm{mM} \mathrm{CHX}$ for $0,4,8$, and $12 \mathrm{~h}$, and cell lysates were collected separately and analyzed for PD-L1 protein levels. $\mathbf{g}$ Influence of WSX1 on protein levels of $\mathrm{p}$-GSK3 $\beta^{\mathrm{Ser} 9}$, total GSK3 $\beta$, $\mathrm{p}-\beta$-cateninSer33/Ser37/Thr41, and total $\beta$-catenin. $\beta$-catenin was directly phosphorylated by GSK3 $\beta$ at Ser33/

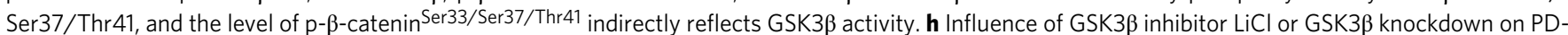
L1 expression. Cell surface PD-L1 expression on WSX1-overexpressing 449WSX1 and 475 WSX1 cells was analyzed by flow cytometry after treatment of LiCl or transfection of GSK3 $\beta$ shRNAs for $48 \mathrm{~h}$. i Statistical analysis results showing that both $\mathrm{LiCl}$ treatment $(P=0.0135 \mathrm{in} 449 \mathrm{WSX} 1$ and $P=0.0052$ in $\left.475^{W S X 1}\right)$ and GSK3 $\beta$ knockdown $\left(P=0.0020\right.$ in $449 \mathrm{WSX}^{\mathrm{W}}$ and $P=0.0015$ in $\left.475 \mathrm{WSX} 1\right)$ increased the proportion of PD-L $1^{+} \mathrm{HCC}$ cells $(n=5$ independent experiments). $\mathbf{j}$ Schematic of site-directed mutation of the consensus motif on PD-L1-NP (S176A, T180A, and S184A), which could not be phosphorylated by GSK3 $\beta$. k SNU449 and SNU475 cells transfected with PD-L1-wild type (WT) or PD-L1-NP (mutated) alone or co-transfected with WSX1. Alterations in cell surface PD-L1 expression levels were determined by flow cytometry. I Quantification of PD-L1 MFI ratio. Site-directed mutation in PD-L1-NP impaired WSX1-mediated PD-L1 downregulation in both SNU449 $(n=3$ independent experiments, $P=0.0066$ compared to PD-L1-WT) and SNU475 cells $(P=$ 0.0004 compared to PD-L1-WT). Quantitative data are presented as mean \pm SD and analyzed by one-way ANOVA or Student $t$ test. Tukey-Kramer multiple comparison test was used for pairwise comparisons in the ANOVA analysis. Unless otherwise noted, data and images shown are representative of 3 independent experiments. All statistical tests were two-sided. ${ }^{\star} P<0.05,{ }^{\star \star} P<0.01,{ }^{\star \star \star} P<0.001$. CHX cycloheximide, SP signal peptide, TM transmembrane domain, ECD extracellular domain, ICD intracellular domain, MFI mean fluorescence intensity. The gating strategy for sorting PD-L1+ HCC cells is shown in Supplementary Fig. 8b. Source data are provided as a Source Data file.

plasmids encoding FLAG-tagged PD-L1, which was not regulated by the endogenous PD-L1 promoter. As expected, WSX1 was capable of downregulating exogenous PD-L1 (Fig. 6b). Our notion was further confirmed by addition of the proteasome inhibitor MG132, which significantly restored WSX1-mediated PD-L1 downregulation in both WSX1-overexpressing cell lines, $449^{\mathrm{WSX} 1}(P=0.0027)$ and $475^{\mathrm{WSX} 1}(P=0.0362$, Fig. 6c, d). Previous studies reported that E3 ubiquitin ligase-mediated lysine 48 (K48) polyubiquitination and subsequent proteasomal degradation control turnover of multiple proteins, including PD-L1 ${ }^{36}$. Consistently, increased K48 ubiquitination of PD-L1 was detected in WSX1-overexpressing cells in the presence of MG132 (Fig. 6e). Moreover, the results of a pulse-chase assay revealed that WSX1 markedly reduced the half-life of the PD-L1 protein (Fig. 6f), supporting our hypothesis that WSX1 destabilizes PD-L1 through promoting its ubiquitin-mediated proteasomal degradation.

Glycogen synthase kinase $3 \beta$ (GSK $3 \beta$ ) is an essential kinase that facilitates PD-L1 phosphorylation and subsequent K48 ubiquitination $^{37}$. Considering that WSX1 promoted PD-L1 ubiquitination, we investigated the connection between WSX1 and GSK3 $\beta$ in terms of PD-L1 degradation. In support of this connection, a previous report ${ }^{38}$ found that both HCC cell lines SNU449 and SNU475, with low endogenous levels of WSX1, had an elevated basal level of phosphorylated GSK3 $\beta$ (p-GSK3 $\beta^{\text {ser9}}$ ), representing inhibition of GSK3 $\beta$ kinase activity. In our study, WSX1 overexpression in SNU449 and SNU475 cells resulted in a dramatic decrease of $\mathrm{p}$-GSK $3 \beta^{\text {ser9 }}$ without a significant effect on total GSK3 $\beta$ levels (Fig. 6g), suggesting an increase in GSK3 $\beta$ activity. GSK3 $\beta$ was reported to specifically phosphorylate $\beta$ catenin at Ser33/Ser37/Thr41 (p- $\beta$-catenin $\left.{ }^{\text {Ser33/Ser37/Thr41 }}\right)^{39}$, whose level indirectly reflects GSK $3 \beta$ enzymatic activity. Notably, an increased amount of $\mathrm{p}-\beta$-catenin $\operatorname{Ser} 33 / \mathrm{Ser} 37 / \mathrm{Thr} 41$ was found as well, supporting our notion that WSX1 enhances GSK3 $\beta$ activity (Fig. 6g). It was reported that the GSK3 $\beta$ inhibitor $\mathrm{LiCl}$ prevented PD-L1 degradation ${ }^{40}$. In our study, treatment with $\mathrm{LiCl}$ substantially rescued WSX1-induced PD-L1 downregulation in $449^{\mathrm{WSX} 1}(P=0.0135)$ and $475^{\mathrm{WSX} 1}$ cells $(P=0.0052$, Fig. $6 \mathrm{~h}, \mathrm{i})$. To further support our hypothesis, short hairpin RNAs against human GSK3 $\beta$ (shGSK3 $\beta$ ) were transfected to establish WSX1knockdown HCC cells. As a result, GSK3 $\beta$ knockdown substantially reversed WSX1-induced PD-L1 downregulation in
$449^{\mathrm{WSX} 1}(P=0.0020)$ and $475^{\mathrm{WSX} 1}$ cells $(P=0.0015$, Fig. $6 \mathrm{~h}, \mathrm{i})$. Furthermore, we synthesized a FLAG-PD-L1-NP expression construct from a wild-type PD-L1 construct (FLAG-PD-L1WT) by mutating 3 phosphorylation consensus motifs on PD-L1 (S176A, T180A, and S184A; Fig. 6j), which was previously reported to completely abolish GSK3 $\beta$-mediated PD-L1 phosphorylation $^{37}$. Consistently, WSX1 prominently downregulated FLAG-PD-L1-WT but only mildly decreased FLAG-PD-L1NP expression (SNU449: $P=0.0066$; SNU475: $P=0.0004$; Fig. 6k, 1). These results illustrated that WSX1 downregulated PD-L1 through facilitating GSK3 $\beta$-mediated PD-L1 phosphorylation and subsequent degradation.

WSX1 enhances GSK3 $\beta$ activity by inactivating the PI3K $\delta$ / AKT signaling pathway. Protein kinase B (PKB/AKT), a serine/ threonine kinase, is reported to phosphorylate GSK3 $\beta$ in vitro and in vivo ${ }^{41,42}$. Furthermore, accumulating evidence has proved that the AKT pathway is critically involved in both hepatocarcinogenesis and PD-L1 regulation ${ }^{2,43}$. We speculated that WSX1 enhances GSK3 $\beta$ activity via regulating AKT. Indeed, we found that WSX1 remarkably inhibited AKT activation, with a decrease of p-AKT ${ }^{\operatorname{ser} 473}$ (Fig. 7a). Tuberin (TSC2) is directly phosphorylated at Thr1462 by activated $\mathrm{AKT}^{44}$. The suppression of AKT activity by WSX1 was further validated by the reduction of $\mathrm{p}$ TSC2 Thr1462 (Fig. 7a).

The results above allowed us to formulate a pathway, in which WSX1 boosts GSK3 $\beta$-mediated PD-L1 degradation through inactivating AKT. To support our notion, we transfected plasmids encoding myristoylated AKT (myr-AKT) to reinvigorate AKT activity in WSX1-overexpressing HCC cells. Indeed, transfection of myr-AKT increased both total AKT and p-AKT ${ }^{\text {ser } 473}$ protein levels (Fig. 7c). More importantly, reinvigoration of AKT activity almost completely reversed WSX1-induced $\mathrm{PD}-\mathrm{L} 1$ reduction in both $449^{\mathrm{WSX} 1}(P<0.0001)$ and $475^{\mathrm{WSX} 1}$ cells $(P<0.000$, Fig. $7 \mathrm{~b}$, c). In support of this in vitro observation, our in vivo study in the HCC mouse model found a negative correlation of WSX1 expression with PD-L1 levels $(r=-0.7802, P=0.0224)$ and AKT activation $(r=-0.8662, P=0.0054)$, as measured by the protein levels of WSX1, PD-L1, and p-AKT ${ }^{\text {ser473 }}$ (Fig. 7d). Together, our results revealed a negative regulatory mechanism involved in the homeostatic control of PD-L1, in which WSX1 prevents 


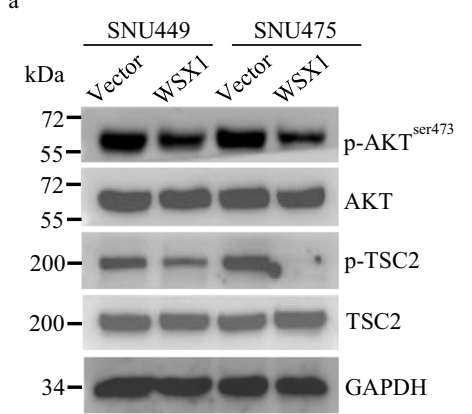

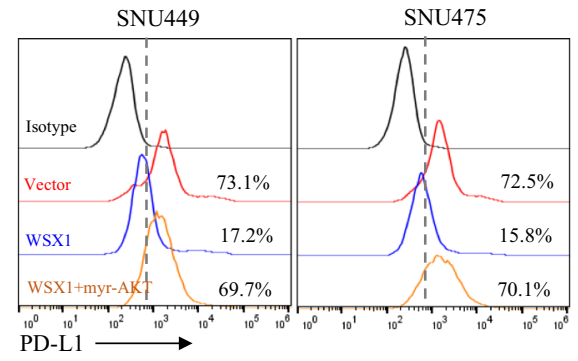
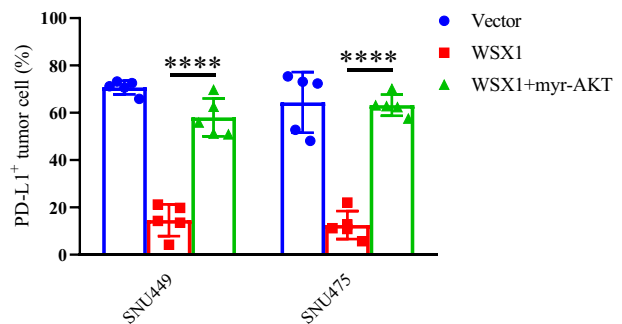
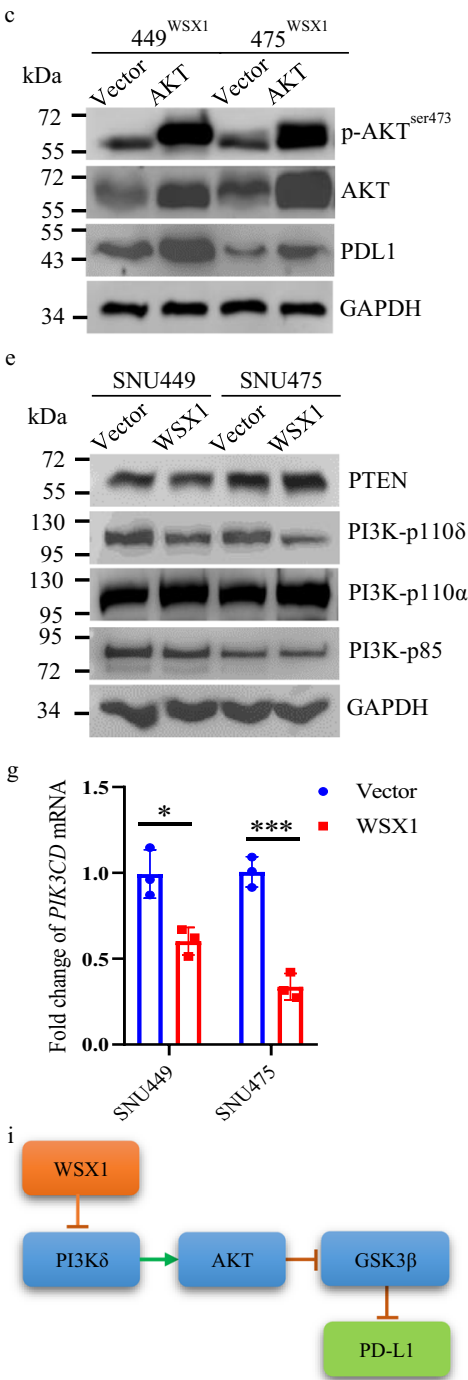

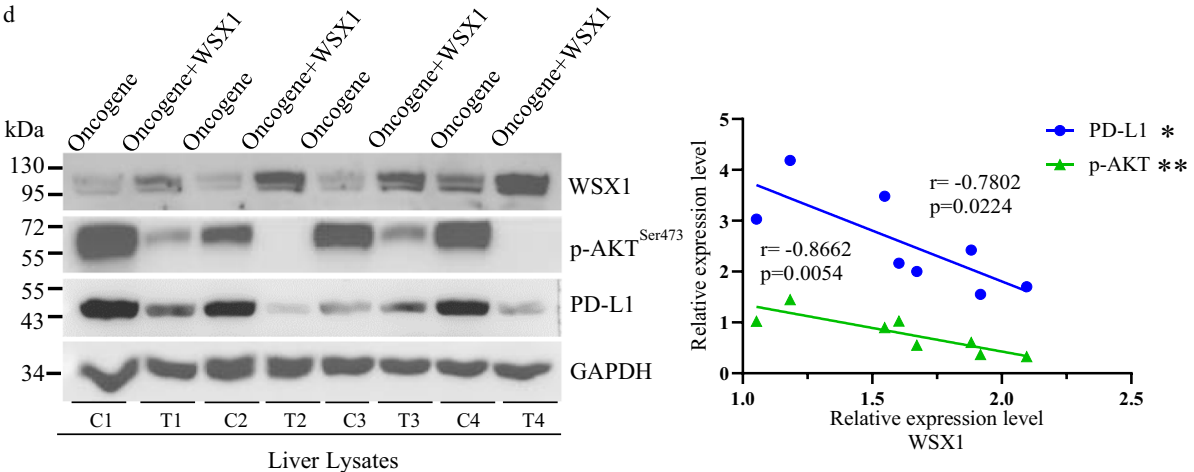

f
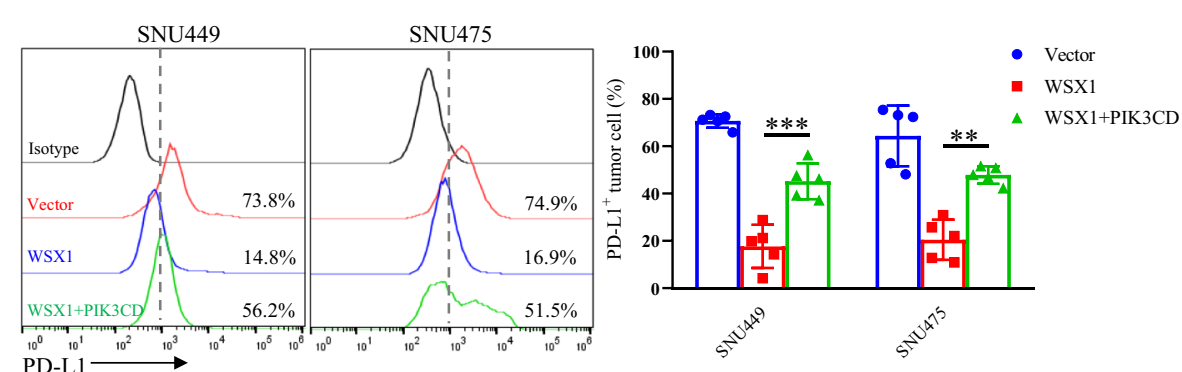

$\mathrm{h}$

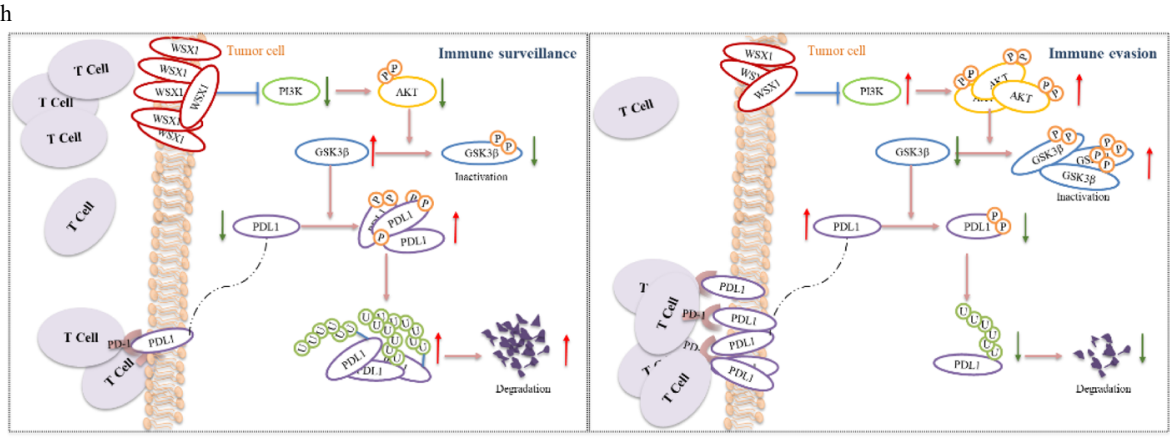

AKT-mediated GSK3 $\beta$ inhibition and thereby activates GSK3 $\beta$ for PD-L1 degradation.

Next, we investigated how WSX1 affects AKT phosphorylation. As reported, the canonical pathway leading to AKT activation is predominantly initiated by activation of phosphatidylinositol 3kinase (PI3K), whose activity can be inhibited by the tumor suppressor PTEN ${ }^{41}$. PI3K encompasses 4 isoforms of the catalytic subunit, known as p110- $\alpha,-\beta$, $-\gamma$, and $-\delta^{45}$. Among them, PI3K $\delta$, one of the PI3K isoforms that constitutively activates the AKT signaling pathway, was recently reported to be highly expressed in HCC and to play significant roles in malignant liver tumors ${ }^{46,47}$. Interestingly, WSX1 had no significant effect on PTEN, PI3K-p85 (PI3K regulatory subunit), or PI3K-p110a (PI3Ka), while remarkably downregulating PI3K-p110 $(\mathrm{PI} 3 \mathrm{~K} \delta$, Fig. 7e). To reveal whether the decrease of
PI3K $\delta$ was responsible for WSX1-induced PD-L1 downregulation, we reintroduced PIK3CD, which encodes PI3K $\delta$, in WSX1overexpressing HCC cells. Our results revealed that, as with the exogenous transfection of myr-AKT, reintroduction of PI3K $\delta$ largely impaired the WSX1-mediated PD-L1 reduction (SNU449: $P=$ 0.0001; SNU475: $P=0.0013$; Fig. 7f). To further explore how WSX1 downregulates PI3K $\delta$, we performed RNA-seq and qRT-PCR analysis in both WSX1-overexpressing and parental HCC cells. Our results consistently showed that WSX1 significantly reduced PIK3CD mRNA levels in both $449^{\mathrm{WSX} 1}(P=0.0139)$ and $475^{\mathrm{WSX} 1}$ cells $(P=0.0006)$, suggesting that WSX1 transcriptionally inhibits the expression of PI3K $\delta$ (Fig. 7g). Consistently, the effect of WSX1 on PI3KS/AKT/GSK3//PD-L1 signaling was also confirmed in WSX1knockdown cells (Supplementary Fig. 7). 
Fig. 7 WSX1 relieves inhibition of GSK3 $\beta$ activity through impeding the PI3Kס/AKT signaling pathway. a Effect of WSX1 overexpression on protein

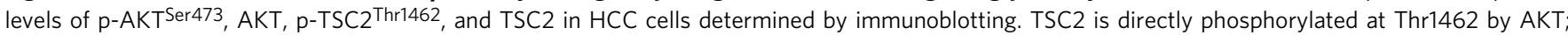
thus p-TSC2 Thr1462 levels indirectly reflect AKT activity. $\mathbf{b}$ Impact of AKT reactivation on WSX1-mediated PD-L1 downregulation detected by flow cytometry. SNU449 and SNU475 cells were transfected with WSX1 alone or co-transfected with membrane-bound myr-AKT. Statistical analysis results are shown on the right ( $n=5$ independent experiments, $P<0.0001$ in both SNU449 and SNU475 cells). c Impact of myr-AKT transfection on protein levels of p-AKTSer473, total AKT, and PD-L1 in 449WSX1 and 475 WSX1 cells. d Immunoblotting analysis of expression of WSX1, PD-L1, and p-AKTSer473 in whole liver lysates obtained from FVB/NJ mice injected with NRAS/AKT oncogenes with or without WSX1 (left, C1-C4 represent independent samples from "oncogene" group, T1-T4 are independent samples from "oncogene + WSX1" group). Statistical analysis of correlations of WSX1 with PD-L1 and pAKT Ser473 expression in mouse livers (right). e Effect of WSX1 on expression of PTEN, PI3K-p85, PI3K-p110 $\alpha$, and PI3K-p1108. f Impact of PIK3CD overexpression on WSX1-mediated PD-L1 reduction (left). PIK3CD encodes PI3K-p1108, which is the key component of PI3K8. Reintroduction of PIK3CD impaired WSX1-induced PD-L1 reduction in both SNU449 ( $n=5$ independent experiments, $P=0.0001)$ and SNU475 cells $(P=0.0013)$. $\mathbf{g}$ Effect of WSX1 on PIK3CD mRNA levels in SNU449 ( $n=3$ independent experiments $P=0.0139)$ and SNU475 HCC cells $(P=0.0006)$. $\mathbf{h}$ A proposed model illustrating

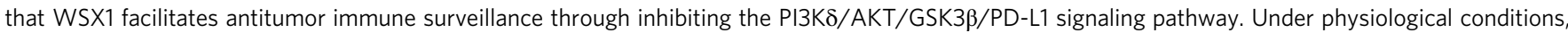
highly expressed WSX1 in hepatocytes transcriptionally downregulates PIK38, thereby reducing AKT activation and subsequently liberating GSK3 $\beta$ kinase activity from inhibition by AKT, leading to boosted GSK3 $\beta$-mediated PD-L1 degradation. Without excessive PD-L1 expression on tumor cells, effector CD8+ T cells maximize their killing effect, resulting in inhibition of HCC development (Left). Otherwise, lack of WSX1 results in uncontrolled neoplastic PD-L1 expression and, ultimately, tumor immune evasion (Right). i Schematic diagram of the interactions among WSX1, PI3K8, AKT, GSK3 3 , and PD-L1.

Quantitative data are presented as mean \pm SD and were analyzed by one-way ANOVA or Student $t$ test. Tukey-Kramer multiple comparison test was used for pairwise comparisons in the ANOVA analysis. Correlation analyses were performed by Pearson correlation test. Unless otherwise noted, data and images shown are representative of 3 independent experiments. All statistical tests were two-sided. ${ }^{\star} P<0.05,{ }^{\star \star} P<0.01,{ }^{\star \star \star} P<0.001$. myr-AKT myristoylated AKT. The gating strategy for sorting PD-L1+ HCC cells is shown in supplementary Fig. 8b. Source data are provided as a Source Data file.

In summary, on the basis of the above results, we are able to reveal an interaction model of WSX1, PI3K $\delta, A K T$, GSK3 $\beta$, and PD-L1 (Fig. 7h, i). Specifically, in physiological conditions, WSX1 is highly expressed in hepatocytes and contributes to the tight control of PD-L1 levels through governing the PI3Kס/AKT/ GSK3 $\beta$ signaling pathway, ensuring a homeostatic PD-L1 expression level on normal hepatocytes, and thereby enabling effective $\mathrm{CD}^{+}{ }^{+} \mathrm{T}$-cell-mediated immunosurveillance. However, when WSX1 is downregulated by multiple oncogenic signals, $\mathrm{PI} 3 \mathrm{~K} \delta$ escapes from WSX1-mediated transcriptional inactivation, resulting in the subsequent activation of AKT. Hyperactive AKT then inactivates GSK3 $\beta$ and thereby blocks GSK3 $\beta$-mediated PDL1 degradation, which results in excessive PD-L1 expression on malignant hepatocytes, leading to $\mathrm{PD}-\mathrm{L} 1 / \mathrm{PD}-1$ axis-mediated tumor immune evasion and, ultimately, HCC development.

\section{Discussion}

Over the past few decades, tumor suppressor genes have been thoroughly investigated for their indispensable role in maintaining genetic integrity in a cell-autonomous manner. These genes can be broadly classified into 2 classes: "gatekeepers," which regulate the cell cycle and replication, and "caretakers," which preserve genetic stability ${ }^{48,49}$. More recently, as more and more researchers have realized the crucial role of the immune system in tumor development ${ }^{5,50}$, ample evidence has suggested that there might be a third class of tumor suppressor gene, "guardians of immune integrity," which ensure effective immunosurveillance ${ }^{51-54}$. Several classical tumor suppressor genes, such as TP53, PTEN, and RB1, have been recently implicated in tumor immunology as a functional extension of their conventional cell-autonomous tumor-suppressive roles. However, none of their tumor-suppressive functions exclusively depend on immunosurveillance ${ }^{51-54}$. In contrast to these dual-function tumor suppressor genes, WSX1's tumor suppressor function exclusively relies on its regulation of adaptive $\mathrm{CD}^{+} \mathrm{T}$-cell immunosurveillance. Neither tumor-cell proliferation (gatekeeper role) nor genetic stability (caretaker role) was significantly affected by WSX1. Instead, WSX1 acts as an "immune surveillance defender," blocking oncogene-induced HCC tumorigenesis in a non-cell-autonomous manner. WSX1 restrained neoplastic PD-L1 expression and protected $\mathrm{CD}^{+} \mathrm{T}$ cell-mediated antitumor immunosurveillance from exhaustion, thus ensuring effective immune elimination of malignant cells.

$\mathrm{T}$-cell exhaustion has a major role in immune dysfunction and tumor immune evasion ${ }^{29}$. Among all tumor-infiltrating lymphocytes, $\mathrm{CD}^{+} \mathrm{T}$ cells are the main subset that performs antitumor immunity through executing $\mathrm{T}$-cell receptor-mediated killing of malignant cells ${ }^{55}$. The correlation of $\mathrm{CD}^{+} \mathrm{T}$-cell infiltration with improved overall survival has been well established ${ }^{55,56}$. Unfortunately, tumor-infiltrating $\mathrm{CD}^{+} \mathrm{T}$ cells are frequently in a state of exhaustion, characterized by a progressive loss of effector functions, robust activation of the T-cell exhaustion driver $\mathrm{TOX}^{31-34}$, and sustained expression of inhibitory receptors such as PD-1, LAG-3, Tim-3, and CTLA-4 ${ }^{28,29}$. Indeed, our results demonstrated severe $\mathrm{CD}^{+}{ }^{+} \mathrm{T}$-cell exhaustion in the NRAS/AKT oncogene-driven spontaneous HCC mouse model. However, WSX1 expression in livers reduced CD8 ${ }^{+}$T-cell dysfunction, evidenced by upregulation of functional markers and downregulation of multiple inhibitory receptors and TOX. Intriguingly, either the use of immune-deficient mice or in vivo depletion of $\mathrm{CD}^{+} \mathrm{T}$ cells completely reversed the tumorsuppressive effect of WSX1, indicating that $\mathrm{CD}^{+}{ }^{+} \mathrm{T}$-cell immunity is indispensable for WSX1-induced HCC suppression. Overall, instead of directly eliminating malignant cells, WSX1 prevented T-cell exhaustion and thus maximized the activity of cytotoxic $\mathrm{CD}^{+}{ }^{+} \mathrm{T}$ cells.

Although T-cell exhaustion has been demonstrated in a variety of human cancers ${ }^{29}$, the underlying mechanisms contributing to its development remain poorly understood. Currently, the intrinsic negative regulatory signaling mediated by inhibitory receptors and the extrinsic suppressive tumor microenvironment are generally accepted as the pivotal pathways driving T-cell exhaustion $^{29}$. In our study, WSX1 injection resulted in a remarkable upregulation of WSX1 on malignant hepatocytes without impacting its expression on infiltrating $\mathrm{CD}^{+} \mathrm{T}$ cells, suggesting that the regulation of T-cell exhaustion by WSX1 is indirect and most likely due to modification of malignant cells. Considerable evidence supports the idea that engagement of PD-L1 on cancer cells with its receptor, PD-1, on effector $\mathrm{T}$ cells is the major mechanism contributing to the exhaustion of tumor-infiltrating lymphocytes and subsequent tumor immune evasion 9,29 . Indeed, our investigation revealed that WSX1 significantly downregulated PD-L1 on HCC cells and 
enhanced $\mathrm{T}$ cell-mediated tumor eradication. In addition to cell surface inhibitory receptors, several immunoregulatory cytokines have been linked to T-cell exhaustion ${ }^{28,29}$. Immunosuppressive cytokines such as IL-10 and TGF- $\beta$ play a positive role, while $\gamma$ chain cytokines, including IL-2, IL-7, and IL-21, have been implicated in antagonizing T-cell dysfunction ${ }^{28,29}$. A more recent study reported that cancer cell-derived cholesterol devitalized $\mathrm{T}$ cells by modulating endoplasmic reticulum stress pathways, highlighting the importance of metabolic factors in immune regulation ${ }^{57}$. Based on our RNA-seq data, no significant alteration of the genes encoding the above immunoregulatory cytokines or metabolites was detected, but their involvement cannot be excluded yet and needs further investigation.

Current immunotherapies targeting the PD-L1/PD-1 axis have exhibited promising clinical responses in multiple tumor types ${ }^{58}$. However, their benefit for overall survival is not satisfactory owing to intrinsic or acquired resistance ${ }^{59}$. Additionally, PD-1 and PD-L1 are located not only on tumor cells but also on normal cells; therefore, nonselective blockade of the PD-L1/PD-1 interaction inevitably causes unfavorable effects on immune homeostasis ${ }^{43}$. In this case, understanding the physiological balance system controlling PD-L1 would benefit the development of more effective therapeutic strategies. Over the past decades, numerous factors, such as IFN- $\gamma$, lactate, and NF- $\mathrm{kB}$, have been found to upregulate PD-L1 expression at translational or posttranslational levels ${ }^{43}$. Considering that the immune system is a tightly regulated network that maintains a delicate and finely tuned balance between immunity and tolerance, there should be a matched control system to restrain immune checkpoints. However, few negative regulators of PD-L1 have been identified. Limited studies claimed that some microRNAs, such as miR-513, could inhibit PD-L1 translation ${ }^{60}$. Recently, a study reported that cyclin D-CDK4 kinase destabilized PD-L1 through upregulating cullin 3-SPOP E3 ligase, which was involved in ubiquitinationmediated PD-L1 degradation ${ }^{61}$. Even so, little is known about the negative control of PD-L1 protein turnover. In this study, we discovered that WSX1 serves as a negative force actively participating in the homeostatic control of PD-L1.

Accumulating evidence demonstrates that PD-L1 is extensively regulated by the ubiquitin/proteasome system ${ }^{43,62,63}$. GSK3 $\beta$, a constitutively activated serine/threonine kinase, was reported to mediate PD-L1 phosphorylation, which facilitated ubiquitin E3 ligase recognition and subsequent ubiquitination of $\mathrm{PD}-\mathrm{L}^{37}$. Activated AKT deactivates GSK3 $\beta^{42,64}$. In our study, we found that WSX1 destabilized PD-L1 protein via downregulating AKT and prompting GSK3 $\beta$-mediated ubiquitination and subsequent proteasomal degradation. Reintroduction of myr-AKT almost completely reversed WSX1-induced PD-L1 reduction. We also noted that either GSK3 $\beta$ knockdown or GSK3 $\beta$ inhibitor LiCl only partially impaired the WSX1-induced effect, indicating that other pathways downstream of AKT might be involved.

Substantial research has shown that AKT is activated in a PI3K-dependent manner ${ }^{41,65}$. PI3K $\delta$, one of the PI3K isoforms, converts phosphatidylinositol (3,4)-bisphosphate (PIP2) lipids to phosphatidylinositol $(3,4,5)$-trisphosphate (PIP3) lipids, which in turn bind to AKT and allow its activation ${ }^{45,66,67}$. PI3K $\delta$ was previously recognized to be typically expressed in cells of hematopoietic origin ${ }^{66}$. However, increasing evidence proved that PI3K $\delta$ was also highly expressed in several solid tumors. PI3K $\delta$ was reported to promote breast and prostate cancer cell proliferation via dampening of PTEN activity and to induce colorectal cancer cell growth and invasion by activating the AKT/ GSK3 $\beta / \beta$-catenin signaling pathway 68,69 . Recently, highly expressed PI3K $\delta$ was also found in HCC and closely correlated with poor survival rates ${ }^{46}$. Interestingly, our results revealed that WSX1 reduced both PI3K $\delta$ mRNA and protein levels and that
PI3K $\delta$ reintroduction largely counteracted WSX1-induced PD-L1 downregulation. Taken together, we reveal a signaling pathway in which WSX1 transcriptionally downregulates PI3K $\delta$ without impacting other classical isoforms of PI3Ks and thereby reduces AKT activity, which in turn prevents AKT-mediated GSK3 $\beta$ inhibition, leading to increased GSK3 $\beta$-mediated PD-L1 degradation. However, as no study has reported a role for WSX1 as a transcription factor, the mechanism underlying the transcriptional inhibitory effect of WSX1 on PI3K $\delta$ needs further investigation.

Notably, chemical AKT inhibitors were reported to inhibit tumor-intrinsic phenotypes and PD-L1 transcription ${ }^{70,71}$, but WSX1 has no significant effect on either PD-L1 mRNA or HCC cell proliferation and migration, indicating a unique regulatory characteristic of WSX1 on AKT/GSK3//PD-L1 signaling that cannot be mimicked by traditional chemical inhibitors. Isoformspecific effects of the individual PI3Ks on AKT activity might be responsible for this difference, at least in part, but the underlying mechanism remains unclear and needs further exploration.

Moreover, in addition to the ubiquitination-mediated proteasomal degradation of PD-L1, it has been reported that glycosylated modification, subcellular transportation, and lysosomal degradation are also closely associated with PD-L1 protein turnover ${ }^{43,72}$. For this reason, further study is required to uncover the overall picture of the functional mechanisms of WSX1.

Collectively, our studies revealed a tumor suppressor gene, WSX1, which functions as a "guardian" of $\mathrm{CD}^{+}{ }^{+} \mathrm{T}$ cell-mediated cancer immunosurveillance and acts as a homeostatic "supervisor" of the immune checkpoint PD-L1. Considering that evidence has illustrated the indispensable role of the liver in host immune homeostasi ${ }^{5}$ - and given the high expression of WSX1 in normal liver tissues and its close interaction with both the critical oncogenic PI3K/AKT signaling pathway and the immune checkpoint PD-L1-the biological function of WSX1 we have uncovered here might only be the tip of the iceberg; the importance of WSX1 might be greater than we thought. Notably, WSX1 is expressed in multiple normal organs besides the liver, including the colon, intestine, and kidney, implying a broader role for WSX1 in immune homeostasis, which might yield insights into the development of more effective immunotherapies.

\section{Methods}

Human tissue microarrays. Our study was approved by the Institutional Review Board of The University of Texas MD Anderson Cancer Center (\#PA12-0604). Human tissue microarrays (FDA662a, BC03116a, and HLiv-HCC180Sur-03) were purchased from Biomax in accordance with a protocol approved by MD Anderson's Institutional Review Board. FDA662a contains 33 types of normal human organ tissue (cerebrum, cerebellum, peripheral nerve, adrenal gland, thyroid gland, spleen, thymus gland, bone marrow, lymph node, tonsil, pancreas, liver, esophagus, stomach, small intestine, colon, lung, salivary gland, larynx, kidney, bladder, testis, prostate, penis, ovary, fallopian tube, breast, endometrium, cervix, cardiac muscle, skeletal muscle, mesothelium, and skin), with samples from each organ taken from 2 individuals. BC03116a contains samples from 40 cases of HCC, 17 normal liver tissues, and 13 NAT. HLiv-HCC180Sur-03 contains samples from 90 cases of HCC and paired NAT with survival follow-up information for 2.0 to 3.7 years. All tissue specimens were obtained from surgical patients of Asian descent from January 2010 to September 2011. No case of overlap was found between BC03116a and HLiv-HCC180Sur-03.

Spontaneous HCC mouse model. Eight- to 10-weeks-old C57BL/6J, FVB/NJ, and NSG mice were purchased from the Jackson Laboratory. WSX1-l- mice with a C57BL/6J background were previously donated by Dr. Frederic de Sauvage (Genentech). IL-27p28 $8^{-1-}$ mice were generated as previously described ${ }^{73}$. All mice were maintained and treated in accordance with ethical guidelines approved by the Institutional Animal Care and Use Committee (IACUC) at MD Anderson.

Because simultaneous activation of AKT/mTOR and RAS/MAPK pathways is often found in human $\mathrm{HCC}^{25}$, previous studies established a spontaneous HCC mouse model based on HDI with plasmids encoding NRASV12/myr-AKT, combined with a transposon system to deliver oncogenes. As previously described $^{25,26}, 20 \mu \mathrm{g}$ of pT3-myr-AKT-HA and pT/Caggs-NRASV12 mixed with sleeping beauty transposase pCMV(CAT)T7-SB100 in a ratio of 25:1 were diluted 
in $2 \mathrm{~mL}$ filtered Dulbecco phosphate buffered saline (DPBS) and then delivered via HDI into the lateral tail vein of mice in 5-7 s at day 0. PiggyBac Dual promoter (PB513B-1) was included as a negative control. Next, a vector plasmid or plasmid encoding WSX1 diluted in $2 \mathrm{~mL}$ DPBS was delivered via HDI weekly. The mice were monitored daily. Due to the aggressive tumor growth, all mice died or were humanely euthanized within 8 weeks after the first injection.

Antibodies and reagents. The following antibodies were used: primary antibody against PD-L1 (Proteintech 17952 and 66248, GeneTex GTX31308), pan-AKT (Cell Signaling Technology 4685), phosphor-AKT 4473 (Abcam ab81283, Cell Signaling Technology 9271), GSK3 $\beta$ (Cell Signaling Technology 12456), phosphorGSK3 $\beta^{\text {Ser9 }}$ (Cell Signaling Technology 5558), WSX1 (Thermo Fisher PA5-96963), $\beta$-catenin (Cell Signaling Technology 8480), phosphor- $\beta$-catenin ${ }^{\text {Ser33/37/Thr41 (Cell }}$ Signaling Technology 9561), PTEN (Cell Signaling Technology 9188), phosphoTSC2 ${ }^{\text {Thr1462 }}$ (Cell Signaling Technology 3617), TSC2 (Cell Signaling Technology 4308), PI3K-p85 (Cell Signaling Technology 4292), PI3K-p110a (Cell Signaling Technology 4255), PI3K-p1108 (Cell Signaling Technology 34050), FLAG (Cell Signaling Technology 2368), Ub (Santa Cruz Biotechnology sc-8017), and WSX1 (clone 237, Monoclonal Antibodies Core Facility at MD Anderson Cancer Center $^{74}$. PerCP/Cyanine5.5 anti-mouse CD3 (BioLegend 100328), V450 antimouse CD8a (Tonbo 75-0081), FITC anti-mouse CD4 (BioLegend 100405), V450 anti-mouse NK1.1 (BD biosciences 560524), PE/Cy7 anti-mouse PD-1 (BioLegend 109109), PE anti-mouse CTLA-4 (BioLegend 106306), PE/Cy7 anti-mouse LAG-3 (BioLegend 125225), PE anti-mouse Tim3 (BioLegend 134009), PE anti-mouse granzyme B (ebioscience 12-8898-80), PE anti-mouse Ki67 (BioLegend 652404), $\mathrm{PE}$ anti-mouse perforin (ebioscience 12-9392-82), PE anti-human CD3 (BioLegend 300308), PE/Cy7 anti-human PD-1 (BioLegend 367414), APC anti-mouse TOX (Miltenyi Biotec 130-118-335), APC anti-mouse IFN- $\gamma$ (ebioscience 17-7311-82), PE anti-mouse IL-2 (BioLegend 503808), PE anti-human WSX1 (R\&D FAB14791P), and HRP anti-human/mouse GAPDH (Proteintech HRP-6000). Anti-mouse CD8a (clone 2.43), anti-mouse CD4 (clone GK1.5), and anti-mouse NK1.1 (clone PK136) for immune cell depletion were constructed and purchased from BioXCell. LiCl, cycloheximide and MG132 were obtained from SigmaAldrich. The dilution of each antibody is shown in Supplementary Table 1.

Cell culture, plasmids, and transfection. Human HCC cell lines SNU398, SNU449, SNU475, HepG2, and Hep3B were purchased from ATCC and were independently validated using short tandem repeat DNA fingerprinting at MD Anderson. Human primary T cells were obtained from Lonza (3W-350). Cells were grown in RPMI 1640 medium supplemented with $10 \%$ fetal bovine serum and $1 \%$ antibiotic mixture

pcDNA3.1-WSX1-FLAG, pcDNA3.1-WSX1-eGFP, and pcDNA3.1-PD-L1FLAG plasmids were obtained from GenScript. The PD-L1-NP mutant (S176A/ T180A/S184) plasmid was generated via site-directed mutagenesis of the pcDNA3.1-PD-L1-FLAG expression vector from GenScript. pT3-myr-AKT-HA was a gift from Xin Chen (Addgene plasmid 31789) ${ }^{75}$. pT/Caggs-NRASV12 was a gift from John Ohlfest (Addgene plasmid 20205) ${ }^{76}$. pCMV(CAT)T7-SB100 was a gift from Zsuzsanna Izsvak (Addgene plasmid 34879) 77. pDONR223-PIK3CD-WT was a gift from Jesse Boehm, William Hahn, and David Root (Addgene plasmid $82222)^{78}$. PiggyBac Dual promoter (PB513B-1) was purchased from System Bioscience. All constructs were confirmed using enzyme digestion and DNA sequencing. All plasmids were purified using the GenElute Endotoxin-Free Plasmid Maxiprep Kit (Sigma) before injecting into mice.

WSX1-stable transfectants in SNU449 and SNU475 cells were generated using a retroviral-based GFP-WSX1 expression system and were selected by FACSAria Cell Sorter (BD Biosciences). For transient transfection, cells were transfected with plasmid DNA using jetPRIME ${ }^{\oplus}$ transfection reagent (Polyplus) or electrotransfection. For GSK3 $\beta$ knockdown, cells were treated with a human GSK3 $\beta$ shRNA expression vector as described in a previous study ${ }^{79}$. The GSK3 $\beta$ shRNA sequence was 5'-GAAAGCTAGATCACTGTAA-3'6. In addition, scrambled shRNAs were added as a negative control (shCtrl). A CRISPR/Cas9 approach was used for WSX1 knockdown. The WSX1 guide RNA sequences (crWSX1: CCTCACCAGAAGGCGGTGTC) were cloned into the px458 vector. The constructs were then transfected into HCC cells using jetPRIME ${ }^{\circledR}$ transfection reagent. Nontargeting crRNAs were added as control (crCtrl).

In vivo $\mathbf{C D 4}^{+} \mathbf{T}$-cell, $\mathbf{C D 8}^{+} \mathbf{T}$-cell, and $\mathbf{N K}$ cell depletion. Depletion antibodies against $\mathrm{CD}^{+} \mathrm{T}$ cells (clone 2.43), $\mathrm{CD} 4^{+} \mathrm{T}$ cells (clone GK1.5), NK cells (clone PK136), or their matched IgG isotypes were administered intraperitoneally into mice twice a week for 3 weeks at a dose of $250 \mu \mathrm{g}$ per mouse, starting on day 3 after the first HDI of NRAS/AKT oncogenes. Efficiency of in vivo immune cell depletion was confirmed by flow cytometry.

T-cell-mediated tumor cell-killing assay. Human primary $\mathrm{T}$ cells were activated with $100 \mathrm{ng} / \mathrm{mL} \mathrm{CD3}$ antibody and $10 \mathrm{ng} / \mathrm{mL} \mathrm{IL-2,} \mathrm{and} \mathrm{then} \mathrm{co-cultured} \mathrm{with}$ human HCC cells in 12-well plates at ratios of 1:1 or 1:2, respectively. After coculture for $48 \mathrm{~h}$, a SYTOX ${ }^{m w}$ AADvanced $^{\mathrm{m}}$ Dead Cell Stain Kit was used to exclude dead cells. Next, the mixed cells were stained with PE-conjugated anti-human
CD3 specific antibodies to distinguish live T-cells and live HCC cells and then evaluated using a BD FACSCanto II cytometer.

Immunohistochemical analysis. Paraffin-embedded human tissue microarrays or mouse liver/tumor tissue sections were deparaffinized, rehydrated, subjected to heat-induced antigen retrieval, blocked in goat serum blocking buffer, and then incubated with primary antibodies overnight at $4{ }^{\circ} \mathrm{C}$. The next day, the sections were washed and then incubated with biotin-conjugated secondary antibodies for $1 \mathrm{~h}$ at room temperature, and then the following detection/visualization kits were used: ABC enhanced Vectastain kit system (Vector Laboratories), DAB peroxidase substrate kit, and hematoxylin and eosin. The images were captured using a Nikon Eclipse Ti microscope and analyzed by ImageJ version 1.8.0.

Cell profiling using CyTOF. Single-cell suspensions of mouse liver were made as described previously ${ }^{80}$. Livers were purfused with cold PBS, mechanically disrupted, and and digested for $20 \mathrm{~min}$ at $37^{\circ} \mathrm{C}$ in Gey's balanced salt solution (GBSS) with $50 \mu \mathrm{g} / \mathrm{ml}$ collagenase IV, then filtered through a $250-\mu \mathrm{m}$ cell strainer. Tumorinfiltrating lymphocytes and hepatocytes were separated using a Ficoll-Paque Plus gradient centrifugation. For CyTOF assay, single T-cells or hepatocytes were incubated with a mixture of metal-labeled antibodies (Supplementary Table 2) for $30 \mathrm{~min}$ at room temperature and then incubated with Cell-ID Intercalator- ${ }^{103} \mathrm{Rh}$ overnight at $4{ }^{\circ} \mathrm{C}$. The labeled samples were detected using a CyTOF 2 mass cytometer (Fluidigm) at the Flow Cytometry and Cellular Imaging Facility at MD Anderson. CyTOF results were analyzed by R package software (R Development Core Team, Version 3.5.3).

Western blotting, co-immunoprecipitation, and pulse-chase assay. For Western blotting analysis, tissues or cells were subjected to lysis in RIPA buffer $(50 \mathrm{mM}$ Tris- $\mathrm{HCl}, \mathrm{pH} 7.4 ; 1 \% \mathrm{NP}-40,0.25 \%$ sodium deoxycholate; $\mathrm{NaCl} 150 \mathrm{mM}$; EDTA 1 $\mathrm{mM}$ ) supplemented with $50 \mathrm{mM} \mathrm{NaF}, 20 \mathrm{mM} \beta$-glycerophosphate, and a complete protease inhibitor cocktail (Roche Diagnostics, Indianapolis, IN, USA). Equal amounts of tissue or cell lysates were separated by $10 \%$ sodium dodecyl sulfatepolyacrylamide gel electrophoresis (SDS-PAGE) and transferred to nitrocellulose membranes by an iBlot gel transfer device (Invitrogen). The membranes were blotted with primary and secondary antibodies to detect the proteins of interest. GAPDH was used as a loading control. For co-immunoprecipitation, cells were lysed in lysis buffer (Tris-HCl $50 \mathrm{mM}, \mathrm{pH} 8.0 ; \mathrm{NaCl} 150 \mathrm{mM}$; EDTA $5 \mathrm{mM} ; 0.5 \%$ NP-40). Lysates were incubated with antibodies on a rotating wheel overnight at $4^{\circ}$ $\mathrm{C}$ and then pulled down using protein $\mathrm{A} / \mathrm{G}$ agarose beads (Pierce) at $4^{\circ} \mathrm{C}$ for $6 \mathrm{~h}$. Beads were collected by centrifugation, washed, and boiled in $2 \times$ SDS-PAGE sample buffers and analyzed by Western blotting. For pulse-chase assay, cells were treated with $25 \mathrm{mM}$ cycloheximide for $0,4,8$, or $12 \mathrm{~h}$, and cell lysates were collected separately and subjected to Western blot analysis to detect PD-L1 protein.

Flow cytometry. Single-cell suspensions were stained with the indicated fluorescence-conjugated primary antibodies for $30 \mathrm{~min}$ at $4{ }^{\circ} \mathrm{C}$ and then analyzed on an Attune acoustic focusing cytometer (Applied Biosystems). For intracellular staining, cells were fixed and permeabilized before incubatind with antibodies. Staining for nuclear transcription factor was performed according to a protocol provided by eBioscience. Stained cells were isolated by flow cytometry and the results were analyzed by FlowJo software. The gating strategies were shown in Supplementary Fig. 8.

qRT-PCR assay. Total RNA was extracted from HCC cells using a RNeasy Plus Mini Kit (QIAGEN, Venlo, Netherlands) according to the manufacturer's instructions and then subjected to complementary DNA synthesis by reverse transcription using a SuperScript III kit (Invitrogen). Quantitative reverse transcriptase PCR (qRT-PCR) assays were performed according to the manufacturer's instructions using a StepOnePlus Real-Time PCR System (Life Technologies). The primers were as follows: $5^{\prime}$-TCACTTGGTAATTCTGGGAGC-3' (PD-L1 forward), 5'-CTTTGAGTTTGTATCTTGGATGCC-3' (PD-L1 reverse), 5'-CATATGTG CTGGGCATTGGC- $3^{\prime}$ (PI3K $\delta$ forward), $5^{\prime}$-TTTCACAGTAGCCCCGGAAC- $3^{\prime}$ (PI3K $\delta$ reverse), 5'-GAGTCAACGGATTTGGTCGT-3' (GAPDH forward), 5'GACAAGCTTCCCGTTCTCAG-3' (GAPDH reverse). All the data analyses were performed using the comparative $\mathrm{Ct}$ method. GAPDH was used as the internal control.

Cell proliferation and migration assay. Cell proliferation was analyzed by Cell Counting Kit-8 (CCK-8) assay as described ${ }^{81,82}$. HCC cells were plated in 96-well plates $\left(4 \times 10^{3}\right.$ cells per well) in DMEM supplemented with $10 \%$ FBS and cultured for indicated time. Next, the CCK8 reagent was added to each well according to the manufacturer's instructions. Results were expressed as the absorbance of each well at $450 \mathrm{~nm}$ as measured using a microplate spectrophotometer (Multiskin spectrum, Thermo Cooperation, America).

Cell migration assays were performed using Transwell chambers (Costar; Corning $)^{83-85}$. Briefly, cells $\left(4 \times 10^{4}\right.$ cells per chamber) were seeded in serum-free medium in the upper chamber, and 10\% FBS was used as chemoattractant in the bottom. Cells were cultured for $24 \mathrm{~h}$. The migrated cells were stained with crystal 
violet and photographed at $\times 200$ magnification. All measurements were performed in triplicates.

Statistical analysis. All quantitative data are presented as mean \pm standard deviation (SD). Statistical significance was determined by two-tailed Student $t$ test or one-way analysis of variance (ANOVA). Tukey-Kramer multiple comparison test was used for pairwise comparisons in the ANOVA analysis. Normality of data was assessed using the Shapiro-Wilk test of normality. Differences in survival curves were analyzed by Kaplan-Meier analysis and the log-rank test. Correlation analyses were performed by Pearson correlation test. All statistical tests were twosided and conducted using GraphPad Prism 8 software (GraphPad Software, La Jolla, CA). Differences were considered significant at $P<0.05$. All data shown are representative of at least 2 independent experiments.

Reporting summary. Further information on research design is available in the Nature Research Reporting Summary linked to this article.

\section{Data availability}

The authors declare that all data supporting the findings of this study are available within the paper and its Supplementary Information files or from the corresponding author upon reasonable request. Source data are provided with this paper.

Received: 6 January 2020; Accepted: 20 May 2021;

Published online: 09 June 2021

\section{References}

1. Siegel, R. L., Miller, K. D. \& Jemal, A. Cancer statistics, 2020. CA Cancer J. Clin. 70, 7-30 (2020)

2. Llovet, J. M. et al. Hepatocellular carcinoma. Nat. Rev. Dis. Prim. 2, 16018 (2016).

3. Villanueva, A. Hepatocellular carcinoma. N. Engl. J. Med. 380, 1450-1462 (2019).

4. Kanda, M., Sugimoto, H. \& Kodera, Y. Genetic and epigenetic aspects of initiation and progression of hepatocellular carcinoma. World J. Gastroenterol. 21, 10584-10597 (2015).

5. Ringelhan, M., Pfister, D., O'Connor, T., Pikarsky, E. \& Heikenwalder, M. The immunology of hepatocellular carcinoma. Nat. Immunol. 19, 222-232 (2018).

6. El-Khoueiry, A. B. et al. Nivolumab in patients with advanced hepatocellular carcinoma (CheckMate 040): an open-label, non-comparative, phase 1/2 dose escalation and expansion trial. Lancet 389, 2492-2502 (2017).

7. Kambhampati, S. et al. Nivolumab in patients with advanced hepatocellular carcinoma and Child-Pugh class B cirrhosis: Safety and clinical outcomes in a retrospective case series. Cancer 125, 3234-3241 (2019).

8. Freeman, G. J. et al. Engagement of the PD-1 immunoinhibitory receptor by a novel B7 family member leads to negative regulation of lymphocyte activation. J. Exp. Med. 192, 1027-1034 (2000).

9. Butte, M. J., Keir, M. E., Phamduy, T. B., Sharpe, A. H. \& Freeman, G. J. Programmed death-1 ligand 1 interacts specifically with the B7-1 costimulatory molecule to inhibit $\mathrm{T}$ cell responses. Immunity 27, 111-122 (2007).

10. Baumeister, S. H., Freeman, G. J., Dranoff, G. \& Sharpe, A. H. Coinhibitory pathways in immunotherapy for cancer. Annu Rev. Immunol. 34, 539-573 (2016).

11. Xiang, X. et al. Prognostic value of PD -L1 expression in patients with primary solid tumors. Oncotarget 9, 5058-5072 (2018).

12. Xie, Q. K. et al. Programmed death ligand 1 as an indicator of pre-existing adaptive immune responses in human hepatocellular carcinoma. Oncoimmunology 5, e1181252 (2016).

13. Kudo, M. Immune checkpoint inhibition in hepatocellular carcinoma: basics and ongoing clinical trials. Oncology 92, 50-62 (2017).

14. Feun, L. G. et al. Phase 2 study of pembrolizumab and circulating biomarkers to predict anticancer response in advanced, unresectable hepatocellular carcinoma. Cancer 125, 3603-3614 (2019).

15. de Jesus, V. H. F. \& Dettino, A. L. A. Update on hepatocellular carcinoma from the 2018 Gastrointestinal Cancer Symposium (ASCO GI). J. Hepatocell. Carcinoma 5, 87-90 (2018)

16. Chen, Q. et al. Development of Th1-type immune responses requires the type I cytokine receptor TCCR. Nature 407, 916-920 (2000).

17. Pflanz, S. et al. WSX-1 and glycoprotein 130 constitute a signal-transducing receptor for IL-27. J. Immunol. 172, 2225-2231 (2004).

18. Yoshida, H. et al. WSX-1 is required for the initiation of Th1 responses and resistance to L. major infection. Immunity 15, 569-578 (2001).
19. Yoshimoto, T. et al. Antiproliferative activity of IL-27 on melanoma. J. Immunol. 180, 6527-6535 (2008).

20. Dibra, D., Cutrera, J. J., Xia, X., Birkenbach, M. P. \& Li, S. Expression of WSX1 in tumors sensitizes IL-27 signaling-independent natural killer cell surveillance. Cancer Res 69, 5505-5513 (2009).

21. Dibra, D., Cutrera, J., Xia, X. \& Li, S. WSX1 expression in tumors induces immune tolerance via suppression of effector immune cells. PLoS One $\mathbf{6}$, e19072 (2011)

22. Dibra, D. et al. Lack of immunomodulatory interleukin-27 enhances oncogenic properties of mutant p53 in vivo. Clin. Cancer Res. 22, 3876-3883 (2016).

23. Yamanaka, A. et al. Hyperproduction of proinflammatory cytokines by WSX 1-deficient NKT cells in concanavalin A-induced hepatitis. J. Immunol. 172, 3590-3596 (2004).

24. Dibra, D. et al. Mutant p53 in concert with an interleukin-27 receptor alpha deficiency causes spontaneous liver inflammation, fibrosis, and steatosis in mice. Hepatology 63, 1000-1012 (2016).

25. Ho, C. et al. AKT (v-akt murine thymoma viral oncogene homolog 1) and NRas (neuroblastoma ras viral oncogene homolog) coactivation in the mouse liver promotes rapid carcinogenesis by way of mTOR (mammalian target of rapamycin complex 1), FOXM1 (forkhead box M1)/SKP2, and c-Myc pathways. Hepatology 55, 833-845 (2012).

26. Liu, Y. T. et al. A novel spontaneous hepatocellular carcinoma mouse model for studying T-cell exhaustion in the tumor microenvironment. $J$. Immunother. Cancer 6, 144 (2018).

27. Batten, M. et al. Interleukin 27 limits autoimmune encephalomyelitis by suppressing the development of interleukin 17-producing T cells. Nat. Immunol. 7, 929-936 (2006).

28. Baitsch, L., et al. Exhaustion of tumor-specific $\mathrm{CD} 8(+) \mathrm{T}$ cells in metastases from melanoma patients. J Clin Invest 121, 2350-2360 (2011).

29. Wherry, E. J. T cell exhaustion. Nat. Immunol. 12, 492-499 (2011).

30. McLane, L. M., Abdel-Hakeem, M. S. \& Wherry, E. J. CD8 T cell exhaustion during chronic viral infection and cancer. Annu Rev. Immunol. 37, 457-495 (2019).

31. Khan, O. et al. TOX transcriptionally and epigenetically programs $\mathrm{CD} 8(+) \mathrm{T}$ cell exhaustion. Nature 571, 211-218 (2019).

32. Scott, A. C. et al. TOX is a critical regulator of tumour-specific $\mathrm{T}$ cell differentiation. Nature 571, 270-274 (2019).

33. Wang, X. et al. TOX promotes the exhaustion of antitumor CD8+ T cells by preventing PD1 degradation in hepatocellular carcinoma. J. Hepatol. 71, 731-741 (2019).

34. Sekine, T., et al. TOX is expressed by exhausted and polyfunctional human effector memory CD8(+) T cells. Sci. Immunol. 5, eaba7918 (2020).

35. Petrovas, C. et al. PD- 1 is a regulator of virus-specific CD8+ T cell survival in HIV infection. J. Exp. Med 203, 2281-2292 (2006).

36. Zhou, M. J., Chen, F. Z. \& Chen, H. C. Ubiquitination involved enzymes and cancer. Med Oncol. 31, 93 (2014).

37. Li, C. W. et al. Glycosylation and stabilization of programmed death ligand-1 suppresses T-cell activity. Nat. Commun. 7, 12632 (2016).

38. Desbois-Mouthon, C. et al. Dysregulation of glycogen synthase kinase-3beta signaling in hepatocellular carcinoma cells. Hepatology 36, 1528-1536 (2002).

39. Yost, C. et al. The axis-inducing activity, stability, and subcellular distribution of beta-catenin is regulated in Xenopus embryos by glycogen synthase kinase 3. Genes Dev. 10, 1443-1454 (1996).

40. Jiang, X. M. et al. Osimertinib (AZD9291) decreases programmed death ligand-1 in EGFR-mutated non-small cell lung cancer cells. Acta Pharm. Sin. 38, 1512-1520 (2017).

41. Manning, B. D. \& Toker, A. AKT/PKB signaling: navigating the network. Cell 169, 381-405 (2017).

42. Cross, D. A., Alessi, D. R., Cohen, P., Andjelkovich, M. \& Hemmings, B. A. Inhibition of glycogen synthase kinase- 3 by insulin mediated by protein kinase B. Nature 378, 785-789 (1995).

43. Wang, Y. et al. Regulation of PD-L1: emerging routes for targeting tumor immune evasion. Front Pharm. 9, 536 (2018).

44. Manning, B. D., Tee, A. R., Logsdon, M. N., Blenis, J. \& Cantley, L. C Identification of the tuberous sclerosis complex-2 tumor suppressor gene product tuberin as a target of the phosphoinositide 3-kinase/akt pathway. Mol. Cell 10, 151-162 (2002).

45. Fruman, D. A. \& Rommel, C. PI3K and cancer: lessons, challenges and opportunities. Nat. Rev. Drug Disco. 13, 140-156 (2014).

46. Ko, E. et al. PI $3 \mathrm{~K} \delta$ is a therapeutic target in hepatocellular carcinoma. Hepatology 68, 2285-2300 (2018).

47. Kang, S., Denley, A., Vanhaesebroeck, B. \& Vogt, P. K. Oncogenic transformation induced by the p110beta, -gamma, and -delta isoforms of class I phosphoinositide 3-kinase. Proc. Natl Acad. Sci. USA 103, 1289-1294 (2006).

48. Kinzler, K. W. \& Vogelstein, B. Cancer-susceptibility genes. Gatekeepers and caretakers. Nature 386, 761, 763 (1997). 
49. Morris, L. G. \& Chan, T. A. Therapeutic targeting of tumor suppressor genes. Cancer 121, 1357-1368 (2015).

50. Jenne, C. N. \& Kubes, P. Immune surveillance by the liver. Nat. Immunol. 14, 996-1006 (2013).

51. Munoz-Fontela, C., Mandinova, A., Aaronson, S. A. \& Lee, S. W. Emerging roles of p53 and other tumour-suppressor genes in immune regulation. Nat. Rev. Immunol. 16, 741-750 (2016).

52. Nicolay, B. N. \& Dyson, N. J. The multiple connections between pRB and cell metabolism. Curr. Opin. Cell Biol. 25, 735-740 (2013).

53. Guo, G. \& Cui, Y. New perspective on targeting the tumor suppressor p53 pathway in the tumor microenvironment to enhance the efficacy of immunotherapy. J. Immunother. Cancer 3, 9 (2015).

54. Li, S. et al. The tumor suppressor PTEN has a critical role in antiviral innate immunity. Nat. Immunol. 17, 241-249 (2016).

55. Flecken, T. et al. Immunodominance and functional alterations of tumorassociated antigen-specific CD8+ T-cell responses in hepatocellular carcinoma. Hepatology 59, 1415-1426 (2014).

56. Durgeau, A., Virk, Y., Corgnac, S. \& Mami-Chouaib, F. Recent advances in targeting CD8 T-cell immunity for more effective cancer immunotherapy. Front Immunol. 9, 14 (2018).

57. Ma, X. et al. Cholesterol induces $\mathrm{CD} 8(+) \mathrm{T}$ cell exhaustion in the tumor microenvironment. Cell Metab. 30, 143-156 e145 (2019).

58. Pardoll, D. M. The blockade of immune checkpoints in cancer immunotherapy. Nat. Rev. Cancer 12, 252-264 (2012).

59. Sharma, P., Hu-Lieskovan, S., Wargo, J. A. \& Ribas, A. Primary, adaptive, and acquired resistance to cancer immunotherapy. Cell 168, 707-723 (2017).

60. Gong, A. Y. et al. MicroRNA-513 regulates B7-H1 translation and is involved in IFN-gamma-induced B7-H1 expression in cholangiocytes. J. Immunol. 182, 1325-1333 (2009).

61. Zhang, J. et al. Cyclin D-CDK4 kinase destabilizes PD-L1 via cullin 3-SPOP to control cancer immune surveillance. Nature 553, 91-95 (2018).

62. Hsu, J. M., Li, C. W., Lai, Y. J. \& Hung, M. C. Posttranslational modifications of PD-L1 and their applications in cancer therapy. Cancer Res. 78, 6349-6353 (2018).

63. Lim, S. O. et al. Deubiquitination and stabilization of PD-L1 by CSN5. Cancer Cell 30, 925-939 (2016).

64. Fang, X. et al. Phosphorylation and inactivation of glycogen synthase kinase 3 by protein kinase A. Proc. Natl Acad. Sci. USA 97, 11960-11965 (2000).

65. Hemmings, B. A. \& Restuccia, D. F. The PI3K-PKB/Akt pathway. Cold Spring Harb Perspect. Biol. 4 a011189 (2012).

66. Vanhaesebroeck, B., Guillermet-Guibert, J., Graupera, M. \& Bilanges, B. The emerging mechanisms of isoform-specific PI3K signalling. Nat. Rev. Mol. Cell Biol. 11, 329-341 (2010).

67. Thorpe, L. M., Yuzugullu, H. \& Zhao, J. J. PI3K in cancer: divergent roles of isoforms, modes of activation and therapeutic targeting. Nat. Rev. Cancer 15, 7-24 (2015).

68. Chen, J. S. et al. PIK3CD induces cell growth and invasion by activating AKT/ GSK-3 $\beta / \beta$-catenin signaling in colorectal cancer. Cancer Sci. 110, 997-1011 (2019).

69. Tzenaki, N. et al. High levels of p1108 PI3K expression in solid tumor cells suppress PTEN activity, generating cellular sensitivity to p $110 \delta$ inhibitors through PTEN activation. FASEB J. 26, 2498-2508 (2012).

70. Simioni, C. et al. The AKT inhibitor MK-2206 is cytotoxic in hepatocarcinoma cells displaying hyperphosphorylated AKT-1 and synergizes with conventional chemotherapy. Oncotarget 4, 1496-1506 (2013).

71. Mittendorf, E. A. et al. PD-L1 expression in triple-negative breast cancer. Cancer Immunol. Res. 2, 361-370 (2014).

72. Burr, M. L. et al. CMTM6 maintains the expression of PD-L1 and regulates anti-tumour immunity. Nature 549, 101-105 (2017).

73. Yan, J. et al. Interleukin-30 (IL27p28) alleviates experimental sepsis by modulating cytokine profile in NKT cells. J. Hepatol. 64, 1128-1136 (2016).

74. Dibra, D. et al. IL27 controls skin tumorigenesis via accumulation of ETARpositive CD11b cells in the pre-malignant skin. Oncotarget 7, 77138-77151 (2016).

75. Calvisi, D. F. et al. Increased lipogenesis, induced by AKT-mTORC1-RPS6 signaling, promotes development of human hepatocellular carcinoma. Gastroenterology 140, 1071-1083 (2011).

76. Wiesner, S. M. et al. De novo induction of genetically engineered brain tumors in mice using plasmid DNA. Cancer Res. 69, 431-439 (2009).

77. Mates, L. et al. Molecular evolution of a novel hyperactive Sleeping Beauty transposase enables robust stable gene transfer in vertebrates. Nat. Genet 41, 753-761 (2009).

78. Kim, E. et al. Systematic functional interrogation of rare cancer variants identifies oncogenic alleles. Cancer Disco. 6, 714-726 (2016).
79. Zhou, W. et al. ShRNA silencing glycogen synthase kinase-3 beta inhibits tumor growth and angiogenesis in pancreatic cancer. Cancer Lett. $\mathbf{3 1 6}$ 178-186 (2012)

80. Blom, K. G. et al. Isolation of murine intrahepatic immune cells employing a modified procedure for mechanical disruption and functional characterization of the B, T and natural killer T cells obtained. Clin. Exp. Immunol. 155, 320-329 (2009).

81. Liu, Y. et al. MSC-derived exosomes promote proliferation and inhibit apoptosis of chondrocytes via lncRNA-KLF3-AS1/miR-206/GIT1 axis in osteoarthritis. Cell Cycle 17, 2411-2422 (2018).

82. Zhang, Z. et al. Upregulated miR-1258 regulates cell cycle and inhibits cell proliferation by directly targeting E2F8 in CRC. Cell Prolif. 51, e12505 (2018).

83. Slevin, M., Kumar, S. \& Gaffney, J. Angiogenic oligosaccharides of hyaluronan induce multiple signaling pathways affecting vascular endothelial cell mitogenic and wound healing responses. J. Biol. Chem. 277, 41046-41059 (2002).

84. $\mathrm{Wu}, \mathrm{M}$. et al. A novel role of low molecular weight hyaluronan in breast cancer metastasis. FASEB J. 29, 1290-1298 (2015).

85. Wu, M. et al. ID1 overexpression promotes HCC progression by amplifying the AURKA/Myc signaling pathway. Int J. Oncol. 57, 845-857 (2020).

\section{Acknowledgements}

This study is supported in part by a grant from the U.S. National Institutes of Health (R01DK102767). We are grateful for the professional support from the MDACC Research Histology Core Laboratory and North Campus Flow Cytometry and Cellular Imaging Core Facility. Editorial support was provided by Dawn Chalaire, Amy Ninetto and Bryan Tutt in Editing Services, Research Medical Library, The University of Texas MD Anderson Cancer Center.

\section{Author contributions}

S.L. and M.W. initiate the original idea and planned the experiments for this work. M.W., X.X. and J.H. perform the experiments. M.W. collects data and perform statistical analyses. M.W. and S.L. prepare the manuscript draft with input from all authors. N.W.F. provides histological evaluation of mice tissues. All authors provide critical feedback and help shaping the research, analysis, and manuscript.

\section{Competing interests}

The authors declare no competing interests.

\section{Additional information}

Supplementary information The online version contains supplementary material available at https://doi.org/10.1038/s41467-021-23864-9.

Correspondence and requests for materials should be addressed to S.L.

Peer review information Nature Communications thanks Christopher Rudd and the other, anonymous, reviewer(s) for their contribution to the peer review of this work. Peer reviewer reports are available.

Reprints and permission information is available at http://www.nature.com/reprints

Publisher's note Springer Nature remains neutral with regard to jurisdictional claims in published maps and institutional affiliations.

Open Access This article is licensed under a Creative Commons Attribution 4.0 International License, which permits use, sharing, adaptation, distribution and reproduction in any medium or format, as long as you give appropriate credit to the original author(s) and the source, provide a link to the Creative Commons license, and indicate if changes were made. The images or other third party material in this article are included in the article's Creative Commons license, unless indicated otherwise in a credit line to the material. If material is not included in the article's Creative Commons license and your intended use is not permitted by statutory regulation or exceeds the permitted use, you will need to obtain permission directly from the copyright holder. To view a copy of this license, visit http://creativecommons.org/ licenses/by/4.0/.

This is a U.S. Government work and not under copyright protection in the US; foreign copyright protection may apply 2021 